\title{
Lithotectonic elements and geological events in the Hengshan-Wutai-Fuping belt: a synthesis and implications for the evolution of the Trans-North China Orogen
}

\author{
GUOCHUN ZHAO*†, ALFRED KRÖNER $\ddagger$, SIMON A. WILDE $\S$, MIN SUN*, \\ SANZHONG LIף, XUPING LI\|, JIAN ZHANG*, XIAOPING XIA* \& YANHONG HE* \\ *Department of Earth Sciences, The University of Hong Kong, Pokfulam Road, Hong Kong \\ $\ddagger$ Institut für Geowissenschaften, Universität Mainz, 55099 Mainz, Germany \\ $\S$ Department of Applied Geology, Curtin University of Technology, GPO Box U1987, Perth 6845, WA, Australia \\ TCollege of Marine Geosciences, Ocean University of China, Qingdao, 266003, China \\ $\|$ College of Geoinformation Science \& Engineering, Shandong University of Science \\ and Technology, Qingdao, 266510, China
}

(Received 3 September 2006; accepted 21 November 2006)

\begin{abstract}
The Hengshan-Wutai-Fuping belt is located in the middle segment of the Trans-North China Orogen, a Palaeoproterozoic continental collisional belt along which the Eastern and Western blocks amalgamated to form the North China Craton. The belt consists of the medium- to highgrade Hengshan and Fuping gneiss complexes and the intervening low- to medium-grade Wutai granite-greenstone terrane, and most igneous rocks in the belt are calc-alkaline and have affinities to magmatic arcs. Previous tectonic models assumed that the Hengshan and Fuping gneiss assemblages were an older basement to the Wutai supracrustal rocks, but recent studies indicate that the three complexes constitute a single, long-lived Neoarchaean to Palaeoproterozoic magmatic arc where the Wutai Complex represents an upper crustal domain, whereas the Hengshan and Fuping gneisses represent the lower crustal components forming the root of the arc. The earliest arc-related magmatism in the belt occurred at 2560-2520 Ma, marked by the emplacement of the Wutai granitoids, which was followed by arc volcanism at 2530-2515 Ma, forming the Wutai greenstones. Extension driven by widespread arc volcanism led to the development of a back-arc basin or a marginal sea, which divided the belt into the Hengshan-Wutai island arc (Japan-type) and the Fuping relict arc. At 2520-2480 Ma, subduction beneath the Hengshan-Wutai island arc caused partial melting of the lower crust to form the Hengshan tonalitic-trondhjemitic-granodioritic (TTG) suites, whereas eastward-directed subduction of the marginal sea led to the reactivation of the Fuping relict arc, where the Fuping tonalitictrondhjemitic-granodioritic suite was emplaced. In the period 2360-2000 Ma, sporadic phases of isolated granitoid magmatism occurred in the Hengshan-Wutai-Fuping region, forming $2360 \mathrm{Ma}, c$. $2250 \mathrm{Ma}$ and 2000-2100 Ma granitoids in the Hengshan Complex, the c. $2100 \mathrm{Ma}$ Wangjiahui and Dawaliang granites in the Wutai Complex, and the 2100-2000 Ma Nanying granitoids in the Fuping Complex. At c. $1920 \mathrm{Ma}$, the Hengshan-Wutai island arc underwent an extensional event, possibly due to the subduction of an oceanic ridge, leading to the emplacement of pre-tectonic gabbroic dykes that were subsequently metamorphosed, together with their host rocks, to form medium- to high-pressure granulites. At 1880-1820 Ma, the Hengshan-Wutai-Fuping arc system was juxtaposed, intensely deformed and metamorphosed during a major and regionally extensive orogenic event, the Lüliang Orogeny, which generated the Trans-North China Orogen through collision of the Eastern and Western blocks. The Hengshan-Wutai-Fuping belt was finally stabilized after emplacement of a mafic dyke swarm at $1780-1750 \mathrm{Ma}$.
\end{abstract}

Keywords: Archaean, Palaeoproterozoic, magmatic arc, subduction, collision, Hengshan-WutaiFuping belt, Trans-North China Orogen.

\section{Introduction}

Major advancements in understanding the geological history of the North China Craton have been made in the past few years, following recognition of a major Palaeoproterozoic orogenic belt, called the TransNorth China Orogen (Fig. 1), which divides the craton into the Eastern and Western blocks (Zhao et al.

†Author for correspondence: gzhao@hkucc.hku.hk
$2001 b$ and references therein). The Trans-North China Orogen is characterized by dominant Neoarchaean to Palaeoproterozoic arc-related juvenile crust with minor reworked basement rocks, fragments of ancient oceanic crust, mélanges, high-pressure granulites, retrograded eclogites, crustal-scale ductile shear zones, and linear fold belts with sheath folds (Li et al. 1990; Li \& Qian, 1991; Zhai, Guo \& Yan, 1992; Wang et al. 1996, 1997; Wu \& Zhong, 1998; Zhao, Cawood \& Lu, 1999; Zhao et al. 2000b; Guo, O’Brien \& Zhai, 2002). These 


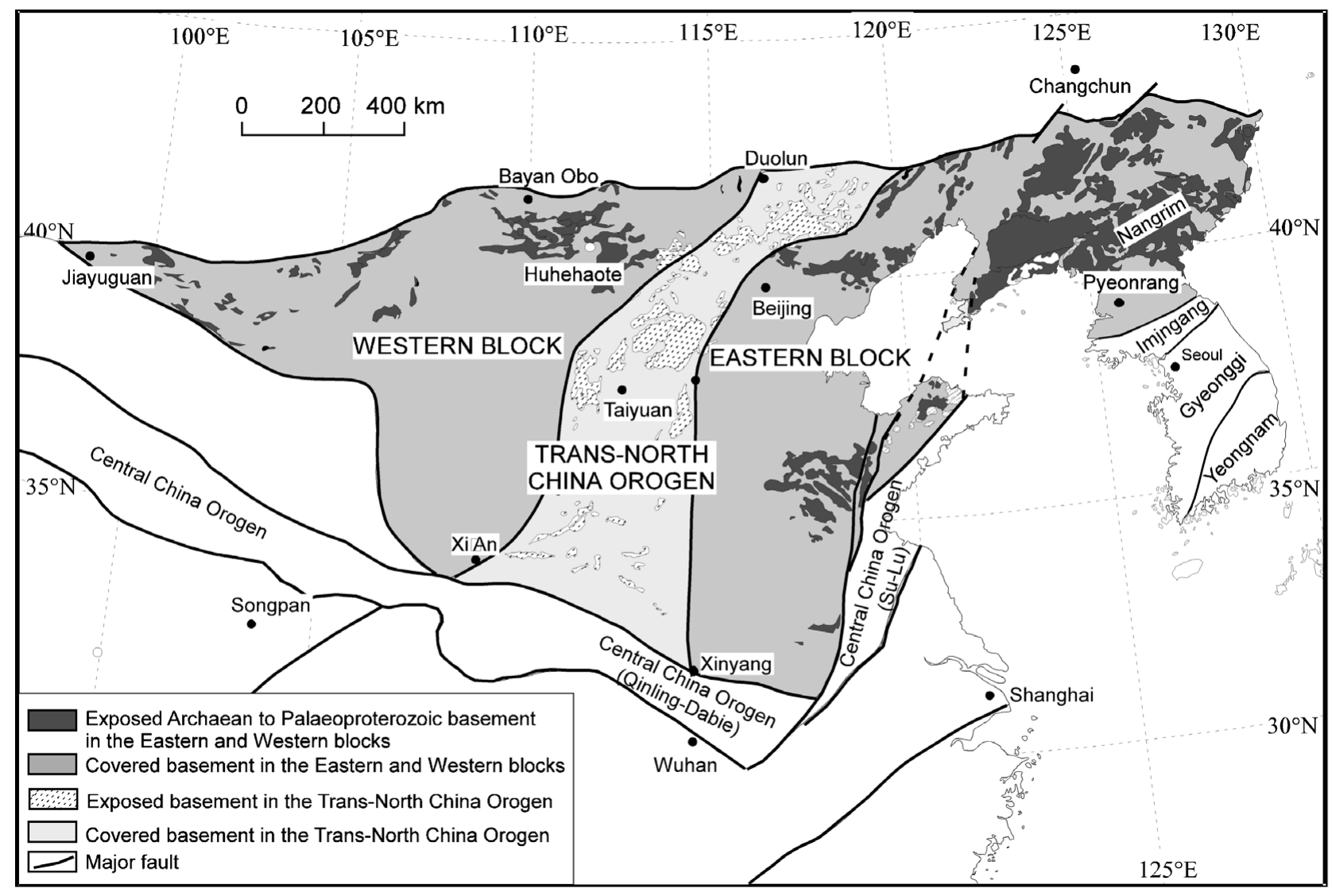

Figure 1. Three-fold tectonic subdivision of the North China Craton proposed by Zhao et al. (1998, 2001b).

lithotectonic elements contrast with the dominant Archaean tonalitic-trondhjemitic-granodioritic (TTG) gneiss domes surrounded by minor supracrustal rocks in the Eastern and Western blocks (Jahn \& Zhang, 1984; Jahn et al. 1987, 1988; Jahn \& Ernst, 1990; Kröner et al. 1998; Zhao et al. 1998; Ge et al. 2003; Wu et al. 2005; Xia et al. 2006a,b). In addition, petrographic and thermobarometric data have revealed a remarkable difference in metamorphic evolution between rocks in the Trans-North China Orogen and the Eastern and Western blocks (Zhao et al. 1998, 1999). The former underwent a major metamorphic event at $c .1 .85$ Ga with clockwise $P-T$ paths involving isothermal decompression and suggesting a collisional environment (Zhao et al. 2000a), whereas the latter experienced a major metamorphic event at c. $2.5 \mathrm{Ga}$, with anticlockwise $P$ $T$ paths involving isobaric cooling, possibly related to underplating of mantle-derived magmas (Zhao et al. 1998, 1999). These differences led Zhao et al. (2001b and references therein) to propose that the Trans-North China Orogen was a continental collisional belt along which the Eastern and Western blocks amalgamated to form the coherent North China Craton at about $1.85 \mathrm{Ga}$ (Zhao, 2001; Zhao et al. 2005 and references therein). According to Zhao (2001), the western margin of the Eastern Block faced a major ocean during Neoarchaean to Early Palaeoproterozoic times, and eastwarddirected subduction of the oceanic lithosphere beneath the western margin of the Eastern Block led to the formation of a magmatic arc that was subsequently incorporated into the Trans-North China Orogen during collision between the Eastern and Western blocks. There is now a coherent outline of timing and tectonic processes involved in the collision between the Eastern and Western blocks (Zhao et al. 1998, 1999; Wu et al. 2000; Wan et al. 2006), but the detailed tectonic architecture and pre-collisional history of the Trans-North China Orogen have not been well constrained, and a number of key geological issues related to the formation and evolution of this orogen are not well resolved. These issues include the relationships between the high-grade gneiss complexes and low-grade granite-greenstone terranes in the orogen, the nature of the magmatic arc system that was subsequently incorporated into the Trans-North China Orogen and the enigma of what magmatic and tectono-metamorphic events took place in the orogen following development of the $2.55 \mathrm{Ga}$ arc and prior to collision of the Eastern and Western blocks at c. $1.85 \mathrm{Ga}$, a time span of around 700 million years.

As the largest and most lithologically representative basement exposure across the Trans-North China Orogen, the Hengshan-Wutai-Fuping belt is the most promising area for investigating the detailed magmatic, depositional, structural and metamorphic history of the orogen. Of particular significance is the presence of a low- to medium-grade granite-greenstone terrane 
(Wutai Complex) intervening between two high-grade gneiss complexes (Hengshan and Fuping complexes). For these reasons, geologists from several countries have carried out extensive geological investigations in the Hengshan-Wutai-Fuping belt and obtained a wealth of new geochronological data and competing interpretations (e.g. Wilde, Cawood \& Wang, 1997, 1998; Wilde, Zhao \& Sun, 2002; Wilde et al. 2004a,b, 2005; Halls, 2000; Kröner, 2002; Kröner et al. 2001, 2005a,b, 2006; Kusky \& Li, 2003; Passchier \& Walte, 2002; Zhao et al. 2002b; Guan et al. 2002; Liu et al. 2000, 2002a,b, 2004a,b, 2005, 2006; Wang et al. 2001, 2003, 2004a,b; O'Brien, Walte \& Li, 2005; P. Peng, unpub. Ph.D. thesis, Chinese Acad. Sciences, Beijing, 2005; Peng et al. 2005; Polat et al. 2005, 2006), which enable resolution of major lithotectonic elements and geological events that occurred in the belt. Here we present a summary and overview of these lithotectonic assemblages and geological events based on field relationships and recent geochronological data, especially SHRIMP U-Pb zircon ages, which provide important insights into understanding the above key issues related to the Neoarchaean to Palaeoproterozoic evolution of the Trans-North China Orogen.

\section{Regional setting}

The North China Craton is a general term used to refer to the Chinese part of the Sino-Korean Platform or Craton. It covers about 1.5 million square kilometres in most of northern China, the southern part of northeastern China, Inner Mongolia, the Bohai Bay and the northern part of the Yellow Sea. The craton is bounded by the Early Palaeozoic Qilianshan Orogen and late Palaeozoic to Mesozoic Central Asian Orogenic Belt to the west and north, respectively, and the Mesozoic Qinling-Dabie and $\mathrm{Su}-\mathrm{Lu}$ ultrahighpressure metamorphic belts to the south and east, respectively (Fig. 1). As mentioned above, the basement of the North China Craton has been divided into the Archaean to Palaeoproterozoic Eastern and Western blocks, separated by the Palaeoproterozoic Trans-North China Orogen (Fig. 1). Lithological, geochemical, structural, metamorphic and geochronological differences between the basement rocks of the Eastern and Western blocks and the Trans-North China Orogen have been summarized by Zhao et al. (2001a) and are not repeated here. Recently, the three-fold subdivision and tectonic model of the North China Craton have been further refined and modified by new structural, petrological and geochronological data obtained over the past few years (Fig. 2; Zhao et al. 2005). These data indicate that the Western Block formed by amalgamation of the Ordos Terrane in the south and the Yinshan Terrane in the north along the EW-trending Khondalite Belt at some time prior to the collision of the Western and Eastern blocks (Fig. 2; Zhao et al. 2005). The data also suggest that the Eastern Block underwent
Palaeoproterozoic rifting along its eastern continental margin in the period 2.2-1.9 Ga, and the closure of this rift basin at c. 1.9 Ga led to the formation of the JiaoLiao-Ji Belt (Fig. 2; Li et al. 2001a,b, 2004a,b, 2005; Hao et al. 2004; Luo et al. 2004; Lu et al. 2006; Zhao et al. 2006). It remains unknown whether oceanic crust formed in the Jiao-Liao-Ji Belt during its rifting stage, though Du, Wang \& Wang (1999) interpreted some ultramafic-mafic rocks as remnants of ophiolites.

The Trans-North China Orogen, a nearly NStrending zone, about $1200 \mathrm{~km}$ long and 100-300 km wide (Fig. 3), is separated from the Eastern and Western blocks by the Xingyang-Kaifeng-ShijiazhuangJiianping Fault and the Huashan-Lishi-DatongDuolun Fault, respectively. Both faults strike NS in the central and southern parts and NE in the north. The presence of voluminous mantle-derived basalts exposed along the two faults suggests that they are deep structures, possibly reaching into the lower crust or upper mantle (Ren, 1980). The main lithotectonic features of the Trans-North China Orogen include: (1) dominant Neoarchaean to Palaeoproterozoic arc-related juvenile crust with minor reworked basement rocks (Kröner et al. 1988; Sun, Armstrong \& Lambert, 1992; Zhao et al. 2000a); (2) linear structural belts defined by strike-slip ductile shear zones, large-scale thrusting and folding, and transcurrent tectonics (Li \& Qian, 1991; Zhang, Dirks \& Passchier, 1994; Zhao et al. 1999); (3) sheath folds and mineral lineations (Wu \& Zhong, 1998); (4) high-pressure granulites and retrograde eclogites (Zhai, Guo \& Yan, 1992; Guo et al. 1999, 2001; Guo, O’Brien \& Zhai, 2002; Guo, Sun \& Zhai, 2005; Guo \& Zhai, 2001; Zhao et al. 2001a; Zhang et al. 2006); (5) clockwise metamorphic $P-T$ paths involving near-isothermal decompression (Liu, Shen \& Geng, 1996; Zhao et al. 2000a; Guo, Sun \& Zhai, 2005); (6) ancient oceanic fragments and ophiolitic mélange (Bai, 1986; Li et al. 1990; Bai, Wang \& Guo, 1992; Wang et al. 1996, 1997; Wu \& Zhong, 1998); (7) synor post-tectonic granites; and (8) post-collisional mafic dyke swarms (Halls et al. 2000; P. Peng, unpub. Ph.D. thesis, Chinese Acad. Sciences, Beijing, 2005; Peng et al. 2005). Most of these lithotectonic elements are classical indicators of collision tectonics.

The Hengshan-Wutai-Fuping belt is situated in the middle segment of the Trans-North China Orogen (Fig. 3) and consists of three distinct tectonic complexes: the upper amphibolite to granulite facies Fuping and Hengshan complexes in the southeast and northwest, respectively, separated by the greenschist- to lower amphibolite-facies Wutai Complex (Fig. 4), which is interpreted as a typical granite-greenstone belt (Bai, 1986; Bai, Wang \& Guo, 1992; Tian, 1991). The Hengshan Complex is bounded in the northwest by the valley of the Sanggan River and is separated from the Wutai Complex by the broad valley of the Hutuo River and thus the nature of the Hengshan-Wutai boundary is unclear. The Fuping Complex was previously considered 


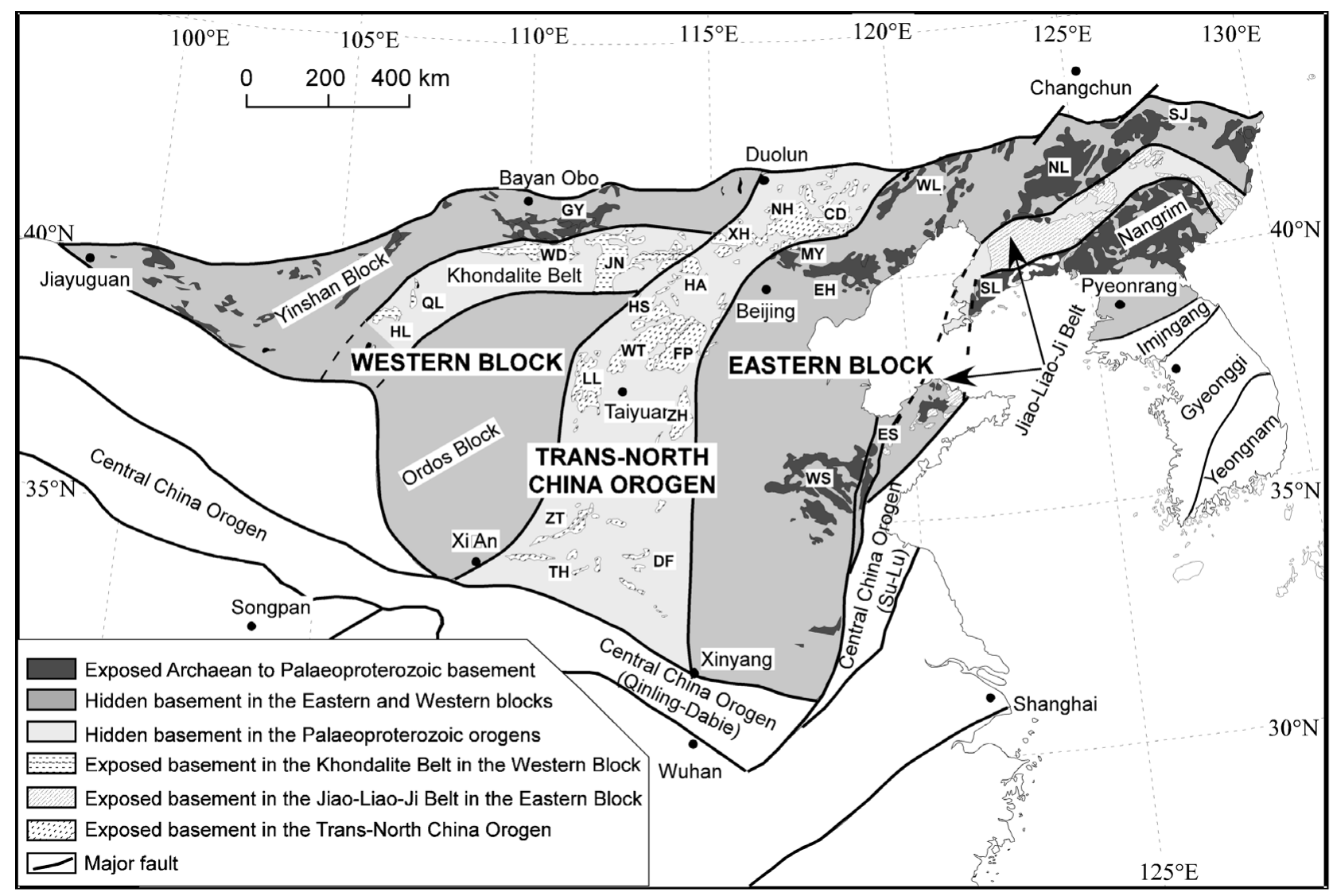

Figure 2. Tectonic subdivision of the North China Craton, modified by Zhao et al. (2005). Abbreviations for metamorphic complexes: CD - Chengde; DF - Dengfeng; EH - Eastern Hebei; ES - Eastern Shandong; FP - Fuping; GY - Guyang; HA - Huai'an; HL - Helanshan; HS - Hengshan; JN - Jining; LL - Lüliang; MY - Miyun; NH - Northern Hebei; NL - Northern Liaoning; QL Qianlishan; SJ - Southern Jilin; SL - Southern Liaoning; TH - Taihua; WD - Wulashan-Daqingshan; WL - Western Liaoning; WS Western Shandong; WT - Wutai; XH - Xuanhua; ZH - Zanhuang; ZT - Zhongtiao.

to be unconformably overlain by the Wutai Complex along the 'Tiebao unconformity' (Bai, 1986; Wu et al. 1989; Tian, 1991; Bai, Wang \& Guo, 1992), but recent research revealed that the so-called 'Tiebao unconformity' is a regional-scale ductile shear zone, named the Longquanguan Ductile Shear Zone by Li \& Qian (1991), and thus the nature of the boundary between the Fuping and Wutai complexes is a tectonic feature.

\section{Major rock units of the Hengshan-Wutai- Fuping belt}

\section{3.a. Hengshan Complex}

The Hengshan Complex is composed of four main rock units (Fig. 4): (1) the Hengshan granitoid gneisses, (2) the Hengshan mafic granulites, (3) the Yixingzhai gneisses, and (4) the Zhujiafang supracrustal assemblage (Tian, 1991; Zhao et al. 2001a).

The Hengshan granitoid gneisses are strongly deformed layered orthogneisses of dioritic-tonalitictrondhjemitic-granodioritic-granitic composition. These rocks are extensively migmatized, and some of the migmatized zones show evidence of in situ melting and advanced anatexis, generating reddish granites. The compositional layering in the gneisses ranges from dark, hornblende-rich dioritic to tonalitic compositions to K-feldspar-dominated leucocratic granitoid varieties. Major element data show that the Hengshan granitoid gneisses belong to a high-alumina calc-alkaline suite (Li \& Qian, 1994), and geochemical data suggest that they were emplaced in a magmatic arc environment (Fig. 5a; Kröner et al. 2005a,b).

Within the Hengshan granitoid gneisses there are discontinuous lenses or layers of amphibolite and highand medium-pressure mafic granulite, ranging from 0.1 to $5 \mathrm{~m}$ in width and 0.1 to $50 \mathrm{~m}$ in length and interpreted as boudinaged gabbroic dykes (Kröner et al. 2005a,b, 2006). In the field, the high-pressure granulite lenses can be distinguished from the surrounding mediumpressure granulites by the presence of coarse-grained textures and lack of brown orthopyroxene in the former. The long axes of the granulite lenses are parallel to the regional foliation of the Hengshan granitoid gneisses. In some places, the interiors of amphibolites and mafic granulites preserve a relict igneous gabbroic/doleritic texture. There can be no doubt that these amphibolites and mafic granulites are remnants of mafic dykes that 


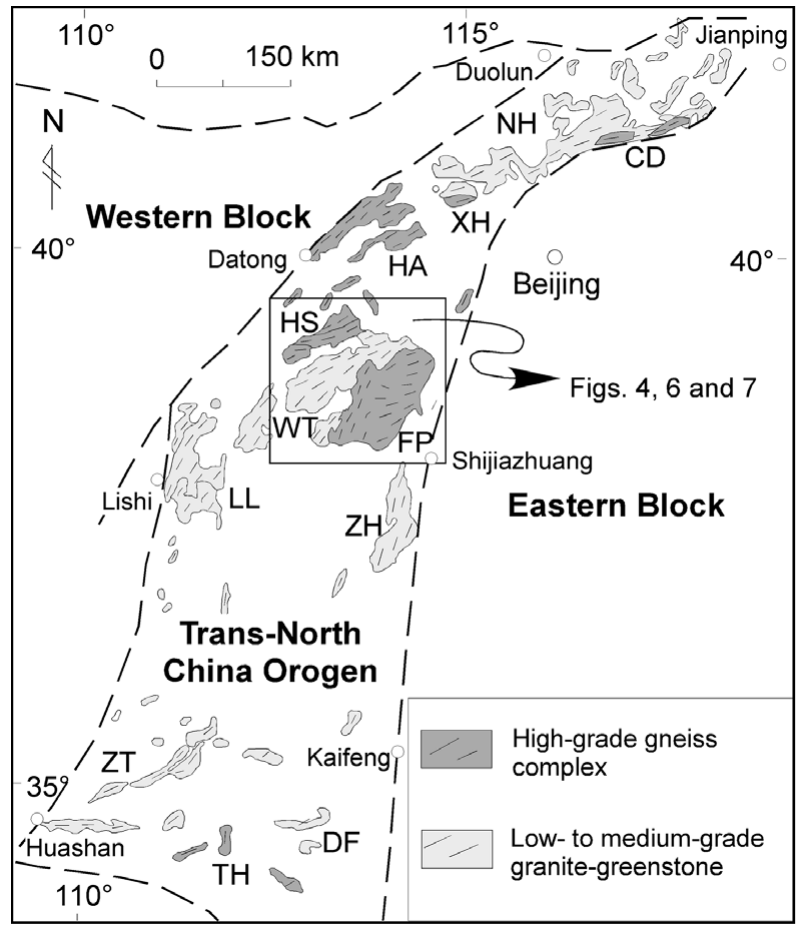

Figure 3. Simplified tectonic map showing the distribution of metamorphic complexes in the Trans-North China Orogen (after Zhao et al. 2000a). Area of Figures 4, 6 and 7 is outlined. Symbols and abbreviations as in Figure 2.

originally intruded into the granitoid rocks, as can still be observed at a few localities in low-strain zones (Kröner et al. 2005a,b, 2006).

The Yixingzhai gneisses occur in the southern part of the Hengshan Complex. They are chemically similar to the Hengshan granitoid gneisses, belonging to a highalumina calc-alkaline suite and forming in a magmatic arc setting (Kröner et al. 2005a,b), but are only metamorphosed to greenschist to lower amphibolite facies and are less deformed, locally preserving igneous textures.

The Zhujiafang assemblage consists predominantly of amphibolite, felsic gneiss, mica schist, banded iron formation (BIF) and quartzite, which occur largely along two nearly EW-trending belts that cut across the regional layering/foliation of the Hengshan granitoid gneisses and Yixingzhai gneisses (Fig. 4). These rocks are characterized by strong ductile deformation and ubiquitous mylonite fabrics and are distinctively lower in metamorphic grade than the other supracrustal rocks within the Hengshan granitoid gneisses. Some Chinese workers have considered the Zhujiafang assemblage to be the equivalent of the Wutai greenstones in the Hengshan area (Tian, 1991; Li \& Qian, 1994).

\section{3.b. Wutai Complex}

The Wutai Complex consists of Neoarchaean to Palaeoproterozoic granitic plutons and metamorphosed volcanic and sedimentary rocks, traditionally named the Wutai and Hutuo 'groups' in the Chinese literature.

The granitoid plutons in the Wutai Complex can be largely divided into three distinct types: the Wutai, Dawaliang and Fengkuangshan granites. The Wutai granites are the major components of the granitoid plutons in the Wutai Complex, including the Chechang-Beitai, Dazhaikou, Duyu, Ekou, Shifu, Lanzhishan, Guangmingshi and Wangjiahui bodies. They are composed of strongly deformed dioritetonalite-trondhjemite-granodiorite suites with penetrative foliations, and thus are considered to be pre-tectonic granitoids (Tian, 1991). Geochemical data suggest that the Wutai granites were emplaced in a magmatic arc environment (Fig. 5a; Liu et al. 2004b, 2005; Kröner et al. 2005b). The Dawaliang-type granites, represented by the Dawaliang body and the younger phase of the Wangjiahui body, are composed of weakly deformed porphyric syenogranites. The Fengkuangshan-type granites, including the Fengkuangshan, Lianhuashan and Pingxingguan bodies, are composed of undeformed A-type granites with a massive structure, interpreted as post-orogenic or anorogenic granites (Tian, 1991).

Based on lithologies and metamorphic grades, the Wutai 'Group' is conventionally subdivided into three subgroups: Shizui, Taihuai and Gaofan subgroups. The Shizui Subgroup consists of peridotite, oceanic tholeiite, dacite, rhyolite, chert, banded iron formation (BIF), sandstone, siltstone, shale, calc-silicate rocks and minor limestone metamorphosed in lower amphibolite facies. Of these, the peridotites, oceanic tholeiites and cherts are considered to represent relict oceanic crust, whereas most sediments are interpreted as continental margin or back-arc basin deposits (Li et al. 1990; Bai \& Dai, 1998; Wu \& Zhong, 1998). The Taihuai Subgroup comprises felsic volcanic rocks and tholeiites metamorphosed in greenschist facies. On the Pearce (1976) major element discrimination diagram (Fig. 5b), most of the felsic and tholeiitic volcanics in the Wutai 'Group' have affinities to modern volcanic-arc assemblages or MORB (Bai, 1986; Wang et al. 2004b). On the $\mathrm{Th} / \mathrm{Ta}$ v. La/Yb diagram (Condie, 1994, 1997, 2005), most of the amphibolite samples from the Wutai 'Group' plot in the arc field, though a few samples are located in the NMORB field and its adjacent areas (Fig. 5c; Wang et al. 2004b). The Gaofan Subgroup contains conglomerates, quartz greywackes, siltstones, limestones, and minor mafic to felsic volcanic rocks metamorphosed from subgreenschist to greenschist facies, which are interpreted as having developed in an intra-arc basin and/or a retro-arc foreland basin (Zhao et al. 2001b). Recent SHRIMP U-Pb zircon ages indicate that the Shizui, Taihuai and Gaofan subgroups are not a single lithostratigraphic sequence, the 'Wutai Group', as previously considered; instead, they are similar-aged volcanic-sedimentary formations that are 


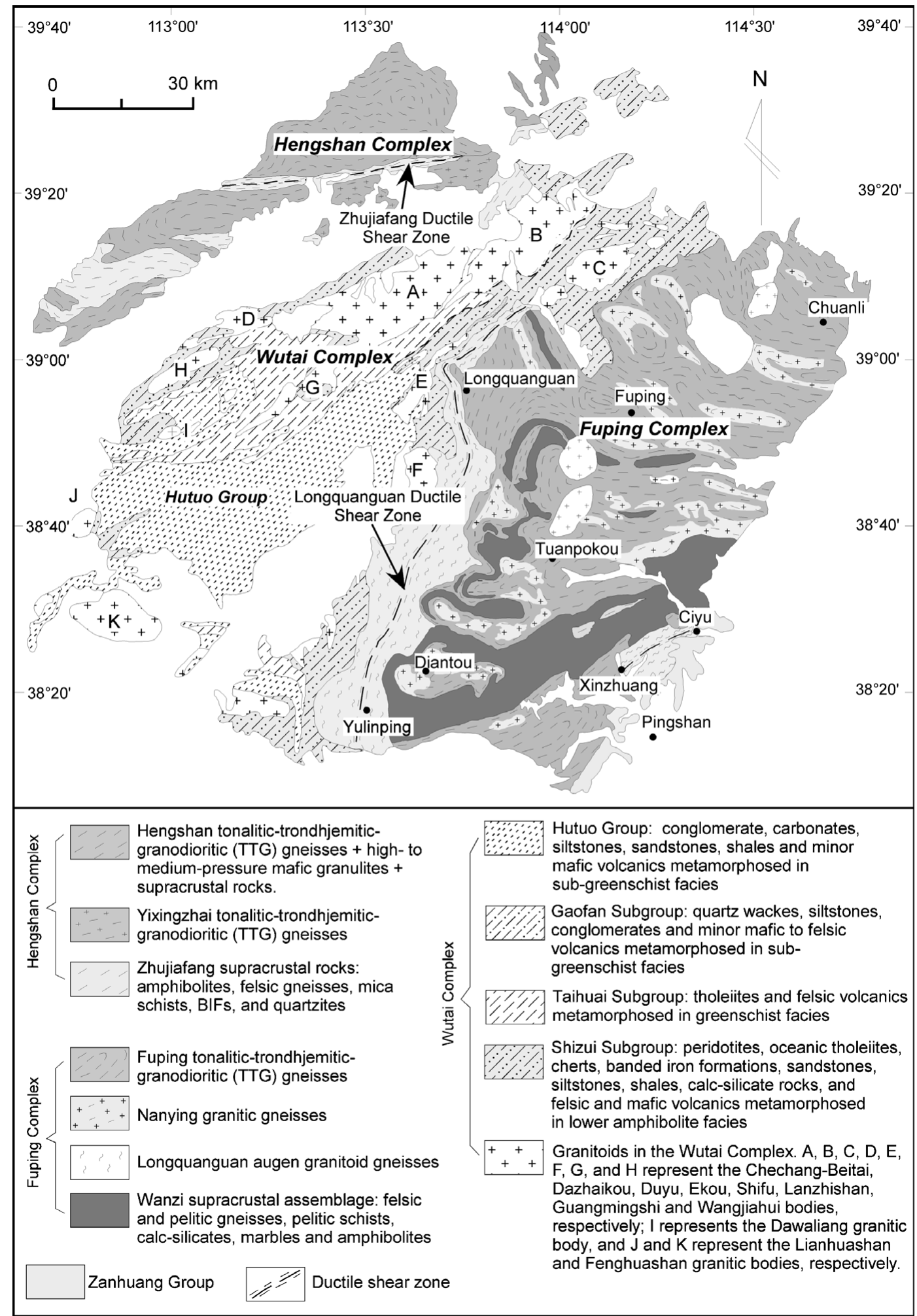

Figure 4. Simplified geological map of the Hengshan, Wutai and Fuping complexes.

structurally disrupted and juxtaposed along a series of NE-SW-trending ductile shear zones (see Section 3.c).

The Hutuo Group is considered to be the youngest unit in the Hengshan-Wutai-Fuping belt and to unconformably overlie the Wutai Group, although in places it is tectonically interleaved with the Wutai sequence. The group ranges upward from an extensive basal sequence of conglomerate into quartzite and other clastic sediments, slate, dolomite and marble, with another unit of conglomerate at the top of the sequence. Rocks of the Hutuo Group are mostly metamorphosed in greenschist facies, although there may be an eastward increase in grade, reaching upper greenschist to amphibolite facies. 
(a)

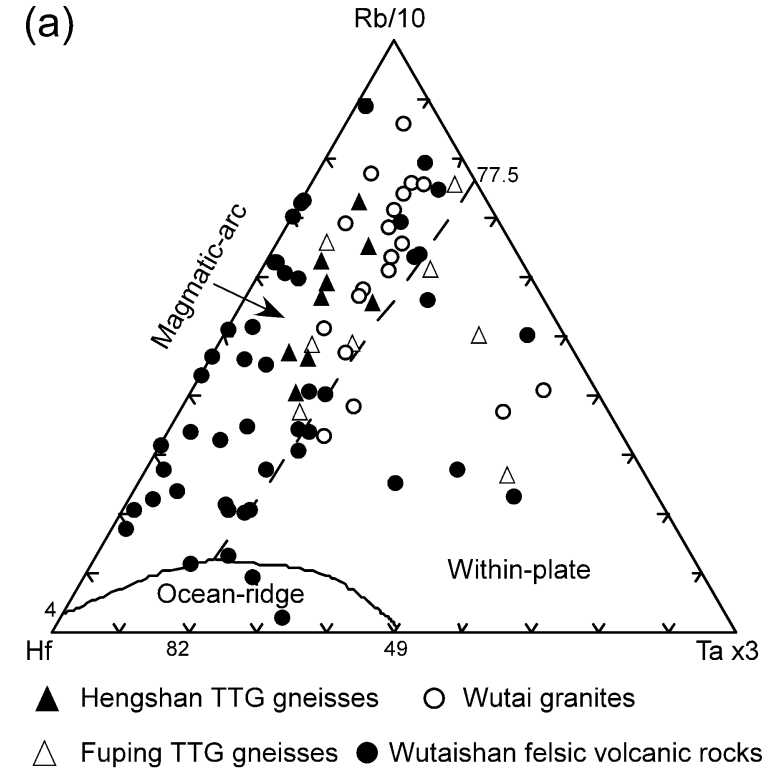

(b)

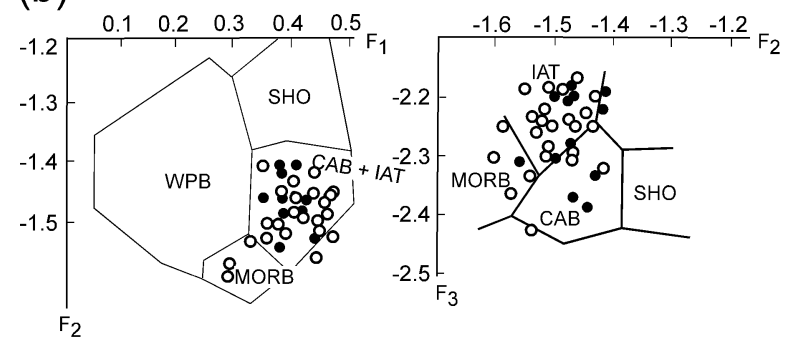

- Amphibolites from the Lower $\square$ Wutai

- Greenschists from the Middle Wutai

$\mathrm{CAB}=$ calc-alkaline basalt; IAT = island arc tholeiite; MORB $=$ mid-ocean ridge basalt;

WPB = within-plate basalt; $\mathrm{SHO}=$ shoshonitic basalt.

(c)

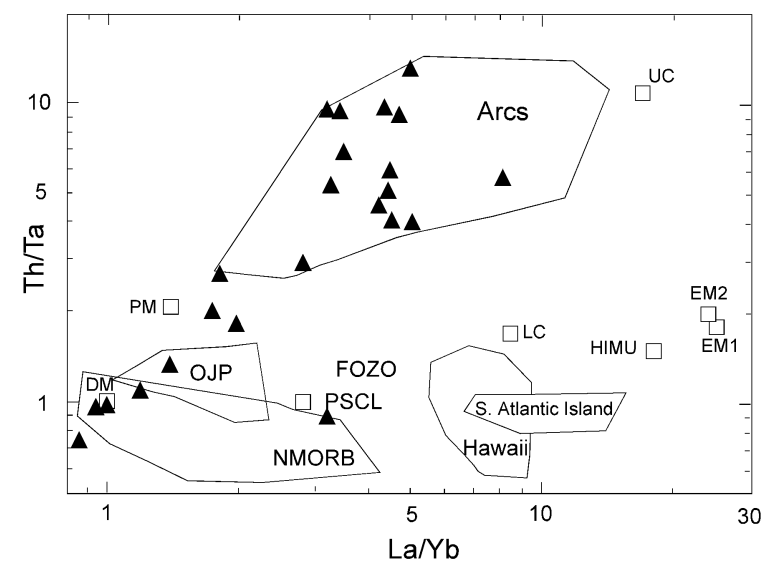

$\mathrm{DM}$, depleted mantle; PM, primitive mantle; PSCL, postArchaean subcontinental lithosphere; LC, lower continental crust; UC, upper continental crust; HIMU, high U/Pb mantle source; EM1 and EM2, enriched mantle sources; FOZO, lower mantle plume component; NMORB, normal mid-ocean ridge basalt; OJP, Ontong-Java Plateau.

Figure 5. (a) $\mathrm{Hf}-\mathrm{Rb} / 10-\mathrm{Ta} \times 3$ discrimination diagram (after Harris, Pearce \& Tindle, 1986) for Hengshan TTG gneisses (Kröner et al. 2005b; Liu et al. 2005), Wutai volcanic and

\section{3.c. Fuping Complex}

The Fuping Complex comprises four distinct rock units, herein called the Fuping granitoid gneisses, Longquanguan augen gneisses, Wanzi supracrustal assemblage, and Nanying granitic gneisses (Fig. 4; Liu et al. 2000; Zhao et al. 2002b).

The Fuping granitoid gneisses make up about $60 \%$ of the complex and consist of medium-grained dioritic, tonalitic, trondhjemitic, granodioritic gneisses enclosing mafic granulite, amphibolite and hornblende gneiss that have undergone a complex history of upper amphibolite- to granulite-facies metamorphism and polyphase deformation. Petrological and geochemical data suggest that, like the Hengshan granitoid gneisses, the Fuping granitoid gneisses formed in a magmatic arc environment (Fig. 5a; Wang, Li \& Liu, 1991).

The Longquanguan augen gneisses are mainly exposed along the Longquanguan ductile shear zone and are composed predominantly of coarse-grained to porphyritic granodioritic and monzogranitic gneisses and mylonitized granitic pegmatites containing K-feldspar phenocrysts, most of which have been intensely deformed to form augen. Enclosed in the Longquanguan augen gneisses are amphibolite and hornblende gneiss enclaves, similar to those in the Fuping granitoid gneisses. The Longquanguan augen gneisses display a tectonic contact with the Wanzi supracrustal assemblage and the Fuping gneisses $(\mathrm{Wu}$ et al. 1989; Li \& Qian, 1991).

The Wanzi supracrustal assemblage forms a $100 \mathrm{~km}$ long, NE-SW-trending belt in the southern part of the complex (15 km wide) that swings northward to the central part of the complex, where it is extensively folded (Fig. 4). The supracrustal rocks are metamorphosed to amphibolite facies and comprise felsic and pelitic gneisses, pelitic schists, calc-silicate rocks, pure and impure marbles and amphibolites (Zhao et al. 2000a,b). Also associated with the supracrustal rocks are several small sillimanitebearing granites which are considered to represent S-type granites derived from partial melting of pelitic gneisses and felsic paragneisses (Zhao et al. 2000a,b).

The Nanying granitic gneisses only occur within the Fuping granitoid gneisses and are dominated by medium- to fine-grained, weakly foliated, magnetitebearing monzogranitic gneiss with minor granodioritic gneiss (Fig. 4; Zhao et al. 2000a,b). In addition to

granitoid rocks (Wang et al. 2004b; Liu et al. 2005) and Fuping TTG gneisses (Liu et al. 2005). (b) Major element discrimination diagram (after Pearce, 1976) for Wutai amphibolites (Lower Wutai) and greenschists (Middle Wutai), based upon functions $F_{1}, F_{2}$ and $F_{3}$ (Bai, Wang \& Guo, 1992). (c) Th/Ta v. La/Yb diagram (Condie, 1994, 1997, 2005) for the greenschist to amphibolite facies basaltic volcanics from the Wutai Complex (Wang et al. 2004b). 
compositional differences, the Nanying gneisses are more massive in structure and more homogeneous in composition than the Fuping granitoid gneisses. In the field where contact relations are preserved, the Nanying granitic gneisses are clearly intrusive into the Fuping granitoid gneisses, but their relatively weak foliation is consistently parallel to the strong, penetrative foliation of the Fuping granitoid gneisses, suggesting that they most likely underwent the same deformational event that resulted in the development of the regional foliation in the Fuping Complex.

\section{Major lithotectonic elements and geological events}

In the last few years, the SHRIMP zircon dating technique, combined with single-grain zircon evaporation and mineral $\mathrm{Sm}-\mathrm{Nd}$ and $\mathrm{Ar} / \mathrm{Ar}$ methods, has been widely applied to date the major rock units of the Hengshan-Wutai-Fuping belt, producing a large number of precise age data for the mountain belt (see references in Table 1). These ages, in conjunction with structural, metamorphic and geochemical considerations, lead us to recognize the following major litho-tectonic assemblages and geological events in the Hengshan-Wutai-Fuping belt.

\section{4.a. Remnants of an old (2.7-2.8 Ga) crustal component}

The oldest known basement components in the Hengshan-Wutai-Fuping belt are rare mediumgrained felsic gneisses that occur interleaved with the younger granitoid gneisses but are difficult to recognize as separate units in the field (Wilde, Cawood \& Wang, 1997; Guan et al. 2002; Kröner et al. 2005a). In the Fuping Complex, xenocrystic zircons from a medium-grained hornblende gneiss enclosed in the Fuping granitoid gneisses were SHRIMP-dated by Guan et al. (2002) at $2708 \pm 8 \mathrm{Ma}$ (Fig. 6, Table 1). A sillimanite paragneiss from the Wanzi Supracrustal assemblage contains two ancient detrital zircons of ages $2827 \pm 8 \mathrm{Ma}$ and $2686 \pm 8 \mathrm{Ma}$, of which the former, as far as we are aware, is the oldest single-grain zircon age so far reported from the Hengshan-WutaiFuping belt (Fig. 6, Table 1). In the Wutai Complex, Wilde, Cawood \& Wang (1997) found four xenocrystic zircons in the $2553 \pm 8 \mathrm{Ma}$ Lanzhishan granites that yielded a weighted mean SHRIMP ${ }^{207} \mathrm{~Pb} / 206 \mathrm{~Pb}$ age of $2702 \pm 10 \mathrm{Ma}$. Wilde (2002) also recognized $2763 \pm 10 \mathrm{Ma}$ and $2660 \pm 7 \mathrm{Ma}$ old xenocrystic zircons in the $2534 \pm 10 \mathrm{Ma}$ Longquanguan augen gneiss (Fig. 6), which is now considered to be part of the Wutai Complex (see Section 4.b). In addition, Wilde et al. (2004a) found two xenocrystic zircons with a weighted mean SHRIMP ${ }^{207} \mathrm{~Pb} /{ }^{206} \mathrm{~Pb}$ age of $2702 \pm 10 \mathrm{Ma}$ from a $2529 \pm 10 \mathrm{Ma}$ meta-andesite of the Zhuangwang Formation. In the Hengshan Complex, a grey trondhjemitic gneiss sample collected from a migmatite domain in Changchenggou ('Great

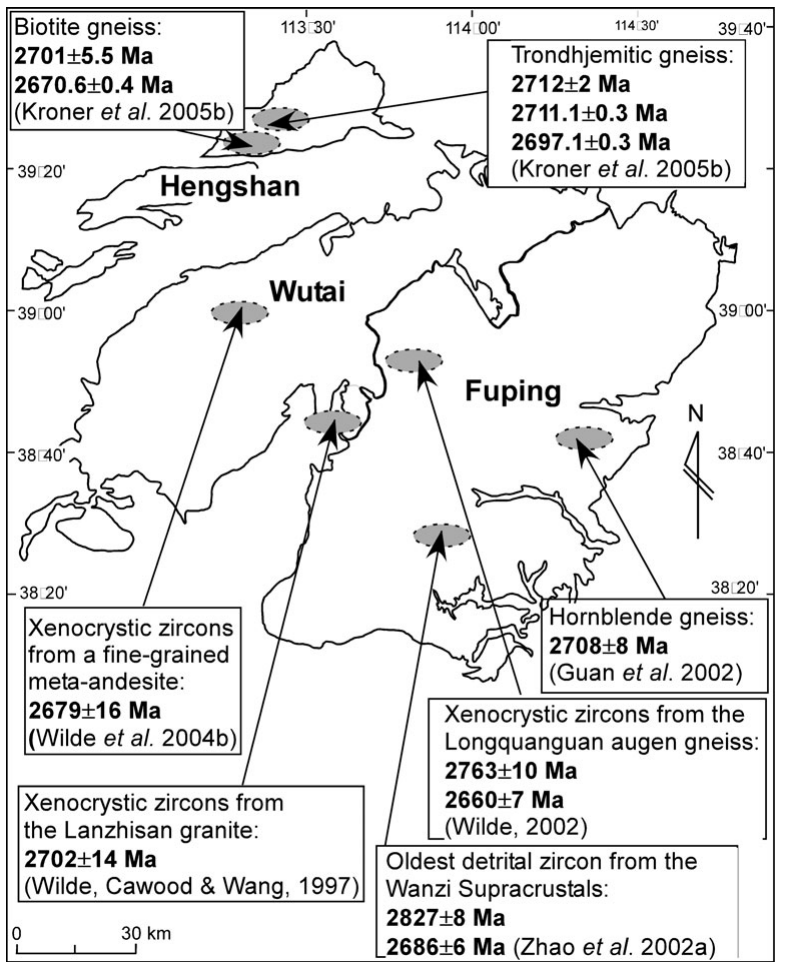

Figure 6. Distribution of 2.7-2.8 Ga xenocrystic zircons or gneisses in the Hengshan-Wutai-Fuping belt.

Wall Valley' in English) and a grey biotite gneiss sample collected from Dashiyu ('Big Stone Valley' in English) were SHRIMP-dated by Kröner et al. (2005b) at $2712 \pm 2 \mathrm{Ma}$ and $2701 \pm 5.5 \mathrm{Ma}$, respectively (Fig. 6). Kröner et al. (2005b) also used the singlegrain evaporation technique to date a foliated grey gneiss from Changchenggou and a fine-grained biotite gneiss from Dashiyu at 2697.1 $\pm 0.3 \mathrm{Ma}$ and $2670.6 \pm 0.4 \mathrm{Ma}$, respectively. Taken together, these data, although sparse, confirm the existence of $c$. $2.7 \mathrm{Ga}$ crustal components in the Hengshan-WutaiFuping region, now tectonically interleaved with the younger gneisses and most probably representing remnants of an older crustal domain whose history and evolution is difficult to reconstruct.

\section{4.b. 2560-2520 Ma: emplacement of Neoarchaean Wutai granites and Longquanguan augen gneisses}

The Wutai granites include the Ekou, Chechang-Beitai, Lanzhishan, Shifu, Guangmingshi and Wangjiahui plutons, of which the oldest is the Ekou pluton dated at $2566 \pm 13 \mathrm{Ma}$ and $2555 \pm 6 \mathrm{Ma}$ (Wilde, Cawood \& Wang, 1997), and the youngest is the Wangjiahui pluton dated at $2517 \pm 12 \mathrm{Ma}$ (Wilde et al. 2005). As the largest exposure of the Wutai granites, the ChechangBeitai pluton is composed of tonalites and granodiorites. Four samples collected from different phases of the pluton yielded SHRIMP zircon ages of $2552 \pm 11$, $2551 \pm 5,2546 \pm 6$ and $2538 \pm 6 \mathrm{Ma}$ (Table 1; Wilde et al. 2005), suggesting that the pluton was emplaced 
Table 1. Summary of isotopic ages for the Hengshan, Wutai and Fuping complexes

\begin{tabular}{|c|c|c|c|c|}
\hline Sample & Description & Age (Ma) & Method & Sources \\
\hline \multicolumn{5}{|c|}{ Ages of remnants of $2.7-2.8 \mathrm{Ga}$ old continental crust } \\
\hline \multirow[t]{2}{*}{990843} & Grey trondhjemitic gneiss (Changchenggou) & $2712 \pm 2$ & SHRIMP & Kröner et al. $(2005 b)$ \\
\hline & Same sample & $2711.1 \pm 0.3$ & EVAP & Kröner et al. $(2005 b$ \\
\hline 990838 & Well foliated grey biotite gneiss (Dashiyu) & $2701 \pm 5.5$ & SHRIMP & Kröner et al. $(2005 b)$ \\
\hline 980811 & Grey granodioritic gneiss (Changchenggou) & $2697.1 \pm 0.3$ & EVAP & Kröner et al. $(2005 b$ \\
\hline 980824 & Finely layered, fine-grained biotite gneiss (Dashiyu) & $2670.6 \pm 0.4$ & EVAP & Kröner et al. $(2005 b)$ \\
\hline \multicolumn{5}{|c|}{ Wutai Complex } \\
\hline $95-\mathrm{PC}-114$ & Xenocrystic zircons from a meta-andesite (Ekou) & $2679 \pm 16$ & SHRIMP & Wilde et al. $(2004 b)$ \\
\hline 95-PC-94 & Xenocrystic zircons from the Lanzhisan granite & $2702 \pm 14$ & SHRIMP & Wilde et al. (1997) \\
\hline \multicolumn{5}{|c|}{ Fuping Complex } \\
\hline \multirow[t]{2}{*}{$95-\mathrm{PC}-65$} & Xenocrystic zircons from the Longquanguan granite & $2763 \pm 10$ & SHRIMP & Wilde (2002) \\
\hline & Same sample & $2660 \pm 7$ & SHRIMP & Wilde (2002) \\
\hline FP50 & Xenocrystic zircons from a biotite gneiss (Diebuan) & $2708 \pm 8$ & SHRIMP & Guan et al. (2002a) \\
\hline \multirow[t]{2}{*}{ FP260 } & Oldest detrital zircons from the Wanzi Supracrustals & $2826 \pm 8$ & SHRIMP & Zhao et al. (2002a) \\
\hline & Same sample & $2686 \pm 6$ & SHRIMP & Zhao et al. $(2002 a)$ \\
\hline \multicolumn{5}{|c|}{ Ages of late Archaean Wutai granitoids and Longquanguan augen gneisses (2560-2520 Ma) } \\
\hline \multicolumn{5}{|c|}{ Ekou Pluton } \\
\hline 95-PC-34 & Deformed, pink, medium-grained granitoid (Ekou) & $2566 \pm 13$ & SHRIMP & Wilde et al. (1997) \\
\hline $95-19$ & deformed, pink, medium-grained granitoid (Ekou) & $2555 \pm 6$ & SHRIMP & Wilde et al. (1997) \\
\hline \multicolumn{5}{|c|}{ Chechang-Beitai pluton } \\
\hline WC7 & Foliated, coarse-grained tonalite (Shahe) & $2552 \pm 11$ & SHRIMP & Wilde et al. (2005) \\
\hline 95-PC-6B & Foliated, coarse-grained tonalite (Taipinggou) & $2551 \pm 5$ & SHRIMP & Wilde et al. (2005) \\
\hline WC6 & Deformed, fine-grained granodiorite (Taipinggou) & $2546 \pm 6$ & SHRIMP & Wilde et al. (2005) \\
\hline WC5 & Deformed, coarse-grained granodiorite (Taipinggou) & $2538 \pm 6$ & SHRIMP & Wilde et al. (2005) \\
\hline \multicolumn{5}{|c|}{ Lanzhishan Pluton } \\
\hline 95-PC-94 & Deformed, coarse-grained granitoid (Changjiagtan) & $2553 \pm 8$ & SHRIMP & Wilde et al. (1997) \\
\hline 95-PC-96 & Deformed, coarse-grained granitoid (Changjiagtan) & $2537 \pm 10$ & SHRIMP & Wilde et al. (1997) \\
\hline \multicolumn{5}{|l|}{ Shifo Pluton } \\
\hline 95-PC-98 & Deformed medium-grained monzogranite (Nanliang) & $2531 \pm 4$ & SHRIMP & Wilde et al. (2005) \\
\hline \multicolumn{5}{|c|}{ Guangmishi Pluton } \\
\hline 95-PC-76 & Deformed, medium-grained granitoid (Guangmishi) & $2531 \pm 5$ & SHRIMP & Wilde et al. (2005) \\
\hline \multicolumn{5}{|c|}{ Wangjiahui Pluton (Grey Phase) } \\
\hline 95-PC-62 & Deformed medium-grained granodiorite (Shi Gang) & $2520 \pm 9$ & SHRIMP & Wilde et al. (2005) \\
\hline $95-\mathrm{PC}-63$ & Deformed medium-grained granodiorite (Shi Gang) & $2517 \pm 12$ & SHRIMP & Wilde et al. (2005) \\
\hline \multicolumn{5}{|c|}{ Longquanguan augen gneisses } \\
\hline WL12 & Sheared porphyritic granitoid (Longquanguan) & $2543 \pm 7$ & SHRIMP & Wilde et al. (1997) \\
\hline WN11 & Sheared tonaltic granitoid (Yushuwan Village) & $2541 \pm 14$ & SHRIMP & Wilde et al. (1997) \\
\hline WL9 & Sheared porphyritic granitoid (Longquanguan) & $2540 \pm 18$ & SHRIMP & Wilde et al. (1997) \\
\hline \multicolumn{5}{|c|}{ Ages of late Archaean Wutai 'Group’ (2530-2515 Ma) } \\
\hline $95 \mathrm{PC}-114$ & Meta-andesite, Zhuangwang 'Formation' (Ekou) & $2529 \pm 10$ & SHRIMP & Wilde et al. $(2004 b)$ \\
\hline 96PC-119 & Meta-andesite, Zhuangwang 'Formation' (Ekou) & $2513 \pm 8$ & SHRIMP & Wilde et al. $(2004 b)$ \\
\hline 95-PC-115 & Meta-andesite, Baizhiyan ‘Formation’ (Ekou) & $2524 \pm 10$ & SHRIMP & Wilde et al. $(2004 b)$ \\
\hline WT13 & Meta-rhyolite, Hongmenyan 'Formation’ (S-T) & $2533 \pm 8$ & SHRIMP & Wilde et al. $(2004 b)$ \\
\hline WT17 & Meta-rhyodacite Hongmenyan 'Formation' (S-T) & $2524 \pm 8$ & SHRIMP & Wilde et al. $(2004 b)$ \\
\hline WT9 & Meta-dacite, Hongmenyan 'Formation' (S-T) & $2523 \pm 9$ & SHRIMP & Wilde et al. $(2004 b)$ \\
\hline WT12 & Meta-rhyodacite, Hongmenyan 'Formation' (S-T) & $2516 \pm 10$ & SHRIMP & Wilde et al. $(2004 b)$ \\
\hline $95-\mathrm{PC}-55 \mathrm{c}$ & Meta-rhyolite, Gaofan 'Subgroup' (Xiazhuang) & $2528 \pm 6$ & SHRIMP & Wilde et al. $(2004 b)$ \\
\hline
\end{tabular}

Ages of late Archaean to Palaeoproterozic Hengshan, Yixingzhai and Fuping TTG gneisses (2520-2480 Ma) Hengshan TTG gneisses

\begin{tabular}{|c|c|}
\hline 980814 & $\begin{array}{l}\text { Dioritic gneiss (Changchenggou) } \\
\text { Same sample }\end{array}$ \\
\hline 990803 & Dioritic gneiss with melt patches (Dashiyu) \\
\hline 990859 & Dioritic gneiss (Xiaoshiyu) \\
\hline & Same sample \\
\hline 980802 & Homogeneous tonalitic gneiss (Dashiyu) \\
\hline 990871 & Tonalitic gneiss, small side valley of Ruyuegou \\
\hline HG1 & Tonalitic gneiss (Dashiyu) \\
\hline HG5 & Garnetiferous trondhjemitic gneiss (Yanmenguan) \\
\hline HG6 & Trondhjemitic gneiss, roadcut near Yanmenguan \\
\hline HG7 & Trondhjemitic gneiss, A roadcut near Yanmenguan \\
\hline 990845 & Foliated trondhjemitic gneiss (Xiaoshiyu) \\
\hline 980838 & Trondhjemitic gneiss (Ruyuegou) \\
\hline 990847 & $\begin{array}{l}\text { Grey, unveined trondhjemitic gneiss (Yanmenguan) } \\
\text { Same sample }\end{array}$ \\
\hline 990854 & Trondhjemitic gneiss (Xiaoshiyu) \\
\hline 980809 & $\begin{array}{l}\text { Foliated pegmatitic red granite-gneiss (Dashiyu) } \\
\text { Same sample }\end{array}$ \\
\hline 980825 & Fine-grained, strained granite-gneiss (Xiaoshiyu) \\
\hline 980833 & Granitic gneiss (Xiaoshiyu) \\
\hline 990873 & $\begin{array}{l}\text { Foliated granite gneiss from migmatite (Guaner) } \\
\text { Same sample }\end{array}$ \\
\hline 980803 & Homogeneous felsic gneiss (Dashiyu) \\
\hline
\end{tabular}

$\begin{array}{cl}2479 \pm 3 & \text { SHRIMP } \\ 2478.2 \pm 0.3 & \text { EVAP } \\ 2475 \pm 2 & \text { SHRIMP } \\ 2506 \pm 5 & \text { SHRIMP } \\ 2504.6 \pm 0.3 & \text { EVAP } \\ 2500.5 \pm 0.3 & \text { EVAP } \\ 2502.3 \pm 0.3 & \text { EVAP } \\ 2520 \pm 15 & \text { SHRIMP } \\ 2520 \pm 10 & \text { SHRIMP } \\ 2526 \pm 12 & \text { SHRIMP } \\ 2507 \pm 4 & \text { SHRIMP } \\ 2504.4 \pm 0.4 & \text { EVAP } \\ 2503.0 \pm 0.3 & \text { EVAP } \\ 2524 \pm 8 & \text { SHRIMP } \\ 2521.7 \pm 0.4 & \text { EVAP } \\ 2504.6 \pm 0.3 & \text { EVAP } \\ 2501 \pm 3 & \text { SHRIMP } \\ 2503.5 \pm 0.3 & \text { EVAP } \\ 2496.3 \pm 0.3 & \text { EVAP } \\ 2492.4 \pm 0.3 & \text { EVAP } \\ 2499 \pm 6 & \text { SHRIMP } \\ 2497.6 & \text { EVAP } \\ 2502.3 \pm 0.6 & \text { EVAP }\end{array}$

Kröner et al. $(2005 b)$ Kröner et al. $(2005 b)$ Kröner et al. $(2005 b)$ Kröner et al. $(2005 b)$ Kröner et al. $(2005 b)$ Kröner et al. (2005b) Kröner et al. (2005b) Wilde et al. (unpub.) Kröner et al. (2005b) Kröner et al. $(2005 b)$ Kröner et al. $(2005 b)$ Kröner et al. (2005b) Kröner et al. (2005b) Kröner et al. (2005b) Kröner et al. (2005b) Kröner et al. (2005b) Kröner et al. (2005b) Kröner et al. $(2005 b)$ Kröner et al. $(2005 b)$ Kröner et al. (2005b) Kröner et al. $(2005 b)$ Kröner et al. $(2005 b)$ Kröner et al. (2005b) 
Table 1. Continued.

\begin{tabular}{|c|c|c|c|c|}
\hline Sample & Description & Age (Ma) & Method & Sources \\
\hline 990821 & Finely layered, fine-grained biotite gneiss (Dashiyu) & $2526 \pm 04.7$ & SHRIMP & Kröner et al. $(2005 b)$ \\
\hline \multicolumn{5}{|c|}{ Yixingzhai TTG gneisses } \\
\hline 96PC153 & Homogeneous tonalitic gneiss, near Yixingzhai & $2513 \pm 15$ & SHRIMP & Wilde (2002) \\
\hline 96РC154 & Quartz dioritic gneiss, a roadcut near Yixingzhai & $2499 \pm 4$ & SHRIMP & Wilde (2002) \\
\hline \multicolumn{5}{|c|}{ Fuping TTG gneisses } \\
\hline FG1 & Layered tonalitic gneiss, a roadcut near Xicaokou & $2523 \pm 14$ & SHRIMP & Zhao et al. (2002a) \\
\hline FP50 & Foliated, hornblende-rich tonalitic gneiss (Diebuan) & $2520 \pm 20$ & SHRIMP & Guan et al. (2002) \\
\hline FP54 & Trondhjemitic gneiss collected from Pingyang & $2513 \pm 12$ & SHRIMP & Guan et al. (2002) \\
\hline FP217 & Trondhjemitic gneiss collected from Tuanpokou & $2499 \pm 9.5$ & SHRIMP & Zhao et al. (2002a) \\
\hline FP216 & Granodioritic gneiss collected from Tuanpokou & $2486 \pm 8$ & SHRIMP & Zhao et al. (2002a) \\
\hline FP08 & Granodioritic gneiss collected from Xicaokou & $2475 \pm 8$ & SHRIMP & Guan et al. (2002) \\
\hline FP236 & Mylonitized monzongranitic gneiss (near Ciyu) & $2510 \pm 22$ & SHRIMP & Zhao et al. (2002a) \\
\hline FP224 & Mylonitized pegmatitic dyke (Xinzhuang) & $2507 \pm 11$ & SHRIMP & Zhao et al. $(2002 a)$ \\
\hline \multirow{2}{*}{\multicolumn{5}{|c|}{$\begin{array}{l}\text { Ages of Palaeoproterozoic (2360-2000 Ma) granitoids } \\
\text { Hengshan Complex }\end{array}$}} \\
\hline & & & & \\
\hline 980806 & Fine-grained granitic orthogneiss (Dashiyu) & $2358.7 \pm 0.5$ & EVAP & Kröner et al. (2005b) \\
\hline 990850 & Layered trondhjemitic gneiss (Dashiyu) & $2329.7 \pm 0.6$ & EVAP & Kröner et al. $(2005 b)$ \\
\hline HG4 & Pegmatitic granite-gneiss (Dashiyu) & $2331 \pm 36$ & SHRIMP & Kröner et al. $(2005 b)$ \\
\hline 980881 & Pegmatite cutting older gneisses (Xiaoshiyu) & $2248.5 \pm 0.5$ & EVAP & Kröner et al. $(2005 b)$ \\
\hline \multirow[t]{2}{*}{980844} & Red anatectic granite (Changchenggou) & $2113 \pm 8$ & SHRIMP & Kröner et al. $(2005 b)$ \\
\hline & Same sample & $2112.3 \pm 0.6$ & EVAP & Kröner et al. $(2005 b)$ \\
\hline \multicolumn{5}{|c|}{ Wutai Complex } \\
\hline D2 & Coarse-grained Dawaliang porphyritic granite & $2176 \pm 12$ & SHRIMP & Wilde (2002) \\
\hline $95-\mathrm{PC}-50$ & Pink Phase of the Wangjiahui granite & $2117 \pm 17$ & SHRIMP & Wilde et al. (2005) \\
\hline 95-PC-51 & Pink Phase of the Wangjiahui granite & $2116 \pm 16$ & SHRIMP & Wilde et al. (2005) \\
\hline $95-\mathrm{PC}-60$ & Pink Phase of the Wangjiahui granite & $2084 \pm 16$ & SHRIMP & Wilde et al. (2005) \\
\hline \multicolumn{5}{|c|}{ Fuping Complex } \\
\hline FP188-2 & Nanying granitoid gneisses, $10 \mathrm{~km}$ south of Fuping & $2077 \pm 13$ & SHRIMP & Zhao et al. (2002a) \\
\hline FP204 & Nanying granitoid gneisses near Dianfang & $2024 \pm 21$ & SHRIMP & Zhao et al. $(2002 a)$ \\
\hline FP30 & Nanying granitoid gneisses collected from Gangnan & $2045 \pm 64$ & SHRIMP & Guan et al. (2002) \\
\hline
\end{tabular}

Ages of Palaeoproterozoic pre-tectonic mafic dykes (now amphiolite or high-pressure granulites)

Hengshan Complex

Ch020902

High-pressure granulite facies mafic dyke (Dashiyu)

$1915 \pm 4$

SHRIMP

$1914 \pm 2 \quad$ SHRIMP

Kröner et al. (2006)

Wutai Complex

High-pressure granulite facies mafic dyke (Dashiyu)

$2147 \pm 5$

SHRIMP

Kröner et al. (2006)

02SX009

Amphibolite facies mafic dyke (Hengling)

Peng (2005)

Ages of the Palaeoproterozoic Hutuo Group and Wanzi supracrustal assemblage

Hutuo Group in the Wutai Complex

$\begin{array}{ll}\text { A younger population from the same sample } & 2180 \pm \pm 9\end{array}$

SHRIMP

SHRIMP

Wanzi Supracrustal assemblage in the Fuping Complex

FP62 A paragneiss collected from Pingyang

FP249 The youngest concordant zircon from a paragneiss

$2097 \pm 46$

$2109 \pm 5$

$\begin{array}{ll}\text { One young discordant zircon from a paragneiss } & 2097 \pm 6 \\ \text { Upper intercept age of igneous zircons cores } & 2099 \pm 22\end{array}$

FP014

Upper intercept age of igneous zircons cores

$2099 \pm 22$

FP037

Upper intercept age of igneous zircons cores

$2112 \pm 23$

SHRIMP

SHRIMP

SHRIMP

LA-ICP

LA-ICP

LA-ICP

Wilde et al. (2004a)

Wilde et al. (2004a)

Guan et al. (2002)

Zhao et al. $(2002 a)$

Zhao et al. $(2002 a)$

Xia et al. $(2006 c)$

Xia et al. $(2006 c)$

Xia et al. $(2006 c)$

Ages of metamorphism of the Hengshan, Wutai and Fuping complexes (1880-1800 Ma)

Hengshan Complex

990803

M068

HG2

One euhedral zircon from a dioritic gneiss (Dashiyu)

Ch990839

Meta-zircons from high-pressure granulite (Dashiyu)

Meta-zircons from high-pressure granulite (Dashiyu)

Coarse-grained pegmatitic melt (Xiaoshiyu)

Same sample

HG1

Ch980871

Ch990853

Ch990886

Meta-zircons from a granitic gneiss (Dashiyu)

Meta-zircons from a retro-eclogite (Xiaoshiyu)

Meta-zircons from a retrograded eclogite (Dashiyu)

Meta-zircons from a mafic granulite (Dashiyu)

Ch990848

Wutai Complex

Unknown

S2010-2-1

S2010-2-1

SZ10

Fuping Complex

FG1

Meta-zircons from high-pressure granulite (Dashiyu)

Garnet amphibolite from the Jingangku Formation

Meta-monazites from a kyanite schist (Jingangku)

Meta-monazites from a kyanite schist (Jingangku)

Meta-monazites from a kyanite schist (Jingangku)

FP217

FP216

FP249

FP260

FP188-2

FP204

Meta-zircons from a tonalitic gneiss (Xicaokou)

Meta-zircons from a trondhjemitic gneiss

Meta-zircons from granodioritic gneiss (Tuanpokou)

One meta-zircon rim from a paragneiss (Jiaan)

One meta-zircon rim from a paragneiss (Diaoyutai)

Meta-zircons from a Nanying granitoid gneisses

Meta-zircons from a Nanying granitoid gneisses
Kröner et al. $(2005 b)$

Kröner et al. (2006)

Kröner et al. (2006)

Kröner et al. (2006)

Kröner et al. (2006)

Kröner et al. (2006)

Kröner et al. (2006)

Kröner et al. (2006)

Kröner et al. (2006)

Kröner et al. (2006)

Wang et al. (2001)

Liu et al. (2006)

Liu et al. (2006)

Liu et al. (2004a)

Zhao et al. (2002a)

Zhao et al. (2002a)

Zhao et al. (2002a)

Zhao et al. (2002a)

Zhao et al. (2002a)

Zhao et al. (2002a)

Zhao et al. (2002a) 
Table 1. Continued.

\begin{tabular}{|c|c|c|c|c|}
\hline Sample & Description & Age (Ma) & Method & Sources \\
\hline FP30 & Two meta-zircon rims from a gneissic granite & $1825 \pm 18$ & SHRIMP & Guan et al. (2002) \\
\hline FP08 & Meta-zircons from a granodioritic gneiss (Xicaokou) & $1817 \pm 26$ & SHRIMP & Guan et al. (2002) \\
\hline FP053 & Meta-zircon rims from a paragneiss (Wuyuezhai) & $1863 \pm 30$ & LA-ICP & Xia et al. $(2006 c)$ \\
\hline FP014 & Meta-zircon rims from a paragneiss (Tuanpokou) & $1822 \pm 20$ & LA-ICP & Xia et al. $(2006 c)$ \\
\hline \multicolumn{5}{|c|}{$\begin{array}{l}\text { Ages of Post-orogenic (1780-1750 Ma) mafic dyke swarms } \\
\text { Hengshan Complex }\end{array}$} \\
\hline $\begin{array}{l}\text { GU12 } \\
\text { Wutai Co }\end{array}$ & Unmetamorphosed mafic dyke (Near Tuling) & $1769.1 \pm 2.5$ & SGD & Halls et al. (2000) \\
\hline 03WT08 & Unmetamorphosed diabase dyke (Wutai County) & $1754 \pm 71$ & SGD & Peng (2005) \\
\hline \multicolumn{5}{|c|}{ Fuping Complex (Taihangshan) } \\
\hline $99 J X-16$ & Unmetamorphosed mafic dyke (Canyansi) & $1765.3 \pm 1.1$ & $\mathrm{~W}-\mathrm{Ar}-\mathrm{Ar}$ & Wang et al. $(2004 a)$ \\
\hline $99 \mathrm{JX}-65$ & Unmetamorphosed mafic dyke (Huangbeiping) & $1774.7 \pm 0.7$ & $\mathrm{~W}-\mathrm{Ar}-\mathrm{Ar}$ & Wang et al. $(2004 a)$ \\
\hline 99JX-71 & Unmetamorphosed mafic dyke (Hujian'an) & $1780.7 \pm 0.5$ & $\mathrm{~W}-\mathrm{Ar}-\mathrm{Ar}$ & Wang et al. $(2004 a)$ \\
\hline
\end{tabular}

SHRIMP - Sensitive high resolution ion microprobe; EVAP - Single grain evaporation. EPMA - Electron Probe Microanalysis; LA-ICP LA-ICP-MS; SGD - Single grain dissolution; W-Ar-Ar - Whole rock ${ }^{40} \mathrm{Ar}-{ }^{39} \mathrm{Ar}$. Meta-zircon - metamorphic zircons; S-T - Shahe-Taihuai Road. Note: In Kröner et al. (2005a,b, 2006a), Dashiyu, Xiaoshiyu and Changchenggou were translated into Big (or Large) Stone Valley, Little (or Small) Stone Valley and Great Wall Valley, respectively.

at about 2550-2540 Ma, slightly later than the Ekou pluton. The Lanzhishan pluton is divisible into older and younger phases with SHRIMP U-Pb zircon ages of $2553 \pm 8$ and $2537 \pm 10 \mathrm{Ma}$, respectively (Wilde, Cawood \& Wang, 1997). SHRIMP U-Pb zircon data also reveal that the Guangmingshi and Shifou plutons were simultaneously emplaced at c. $2531 \mathrm{Ma}$ and probably belong to the same pluton. In summary, the above SHRIMP U-Pb zircon ages confirm the endArchaean (2560-2520 Ma) emplacement of the Wutai granites which reflect the earliest arc-related magmatic event in the Hengshan-Wutai-Fuping belt.

The Longquanguan augen gneisses are restricted to the Longquanguan ductile shear zone and were previously interpreted as the youngest lithological unit in the Fuping Complex (Liu et al. 1985). However, recent SHRIMP U-Pb zircon ages reveal that the Longquanguan augen gneisses were emplaced at $c$. 2540 Ma (Wilde, Cawood \& Wang, 1997), broadly simultaneous with the Wutai gneissic granites, but older than the 2520-2480 Ma Fuping TTG gneisses (see Section 4.d). Furthermore, magmatic zircons of the Longquanguan augen gneisses are not only identical in age to those of the Lanzhishan Granite, but both granitoids contain inherited zircons and zircon cores giving ages of c. $2700 \mathrm{Ma}$. Therefore, the Longquanguan augen gneisses most likely represent the equivalents of the Wutai granites but were structurally reworked during the development of the Longquanguan ductile shear zone. Similarities in fabric and petrographic features as well as the close spatial distribution between the Longquanguan augen gneisses and some of the Wutai granites (e.g. Lanzhishan granite) support this conclusion.

\section{4.c. 2530-2515 Ma: formation of the Neoarchaean Wutai 'Group'}

The volcano-sedimentary rocks of the Wutai 'Group' have long been considered to be either older than, or coeval with, the Wutai granites (Tian, 1991; Bai, Wang \&
Guo, 1992). However, SHRIMP U-Pb zircon ages show that the volcanic rocks of the Wutai 'Group' formed at 2530-2515 Ma (Wilde et al. 2004a), later than most of the Wutai granites. Traditionally, the Wutai 'Group' was subdivided into the lower, middle and upper sequences, represented by the Shizui, Taihuai and Gaofan subgroups, respectively (Bai, 1986; Bai, Wang \& Guo, 1992; Tian, 1991). However, SHRIMP zircon ages reveal no difference between volcanic rocks previously considered to occupy different stratigraphic levels within the Wutai 'Group' (Table 1). For example, zircons from a felsic tuff of the Gaofan Subgroup provided a weighted mean ${ }^{207} \mathrm{~Pb} /{ }^{206} \mathrm{~Pb}$ age of $2528 \pm 6 \mathrm{Ma}$, virtually the same as the age of $2529 \pm 10 \mathrm{Ma}$ obtained for a felsic tuff of the Shizui Subgroup (Wilde, 2002). A meta-andesite, collected from the Zhuangwang Formation of the Shizui Subgroup, yielded a SHRIMP U-Pb zircon age of $2513 \pm 8 \mathrm{Ma}$ (Wilde et al. 2005), which is the youngest age so far obtained for the Wutai 'Group'. Five rhyolite or rhyodacite samples collected from the Taihuai Subgroup yielded SHRIMP U-Pb zircon ages ranging from $2533 \pm 8 \mathrm{Ma}$ to $2516 \pm 10 \mathrm{Ma}$ (Wilde et al. 2005), suggesting that their eruption was largely coeval with that of the volcanic rocks in the Shizui and Gaofan subgroups. These results, together with field observations, suggest that the Wutai 'Group' is not a simple lithostratigraphic succession but constitutes a lithotectonic assemblage of volcano-sedimentary rocks that formed in the period 2530-2515 Ma. The volcanic rocks of the Wutai 'Group' have a close affinity to modern volcanic arc assemblages (Bai, 1986; Wang et al. 2004b; Polat et al. 2005) and may thus document the earliest arc-related volcanism in the HengshanWutai-Fuping belt.

\section{4.d. 2520-2480 Ma: emplacement of Neoarchaean to Palaeoproterozoic Hengshan, Yixingzhai and Fuping TTG gneisses}

Granitoid gneisses are the major components of the Hengshan and Fuping complexes, making up 70-80 \% 
of the total exposures of both the complexes (Fig. 4). Conventionally, the high-grade Hengshan and Fuping complexes were assumed to be older than the low-grade Wutai Complex (Tian, 1991; Bai, Wang \& Guo, 1992), and the Hengshan and Fuping TTG gneisses were considered to have been emplaced prior to intrusion of the Wutai granitoids (Tian, 1991; Bai, Wang \& Guo, 1992). However, recent SHRIMP and evaporation zircon dating does not support these assumptions. As shown in Table 1, SHRIMP U-Pb ages for magmatic zircons indicate that the Fuping TTG gneisses were emplaced between 2523 and $2486 \mathrm{Ma}$ (Guan et al. 2002; Zhao et al. 2002b). Similarly, SHRIMP and evaporation zircon ages show that the Hengshan TTG gneisses were also emplaced between 2524 and $2479 \mathrm{Ma}$ (Table 1; Kröner et al. 2005a,b). An important conclusion from these data is that the Hengshan and Fuping tonalitic-trondhjemitic-granodioritic gneisses are younger than the Wutai granitoids; the latter were emplaced during Neoarchaean times, whereas the former were emplaced in the period between Neoarchaean and Early Palaeoproterozoic times. This conclusion contrasts with previous models that assumed the high-grade Fuping and Hengshan complexes to constitute an older basement below the lower grade Wutai Complex.

The amphibolite facies Yingxingzhai TTG gneisses in the southern Hengshan area have long been considered to be younger than the granulite facies Hengshan TTG gneisses in the northern area, but recent SHRIMP zircon ages do not support this assumption. Zircons from two Yingxingzhai TTG gneiss samples were SHRIMP-dated by Wilde (2002) at $2513 \pm 5 \mathrm{Ma}$ and $2499 \pm 4 \mathrm{Ma}$ (Table 1), which are similar to the age range (2526-2480 Ma) of the Hengshan TTG gneisses (Table 1; Kröner et al. 2005a,b). Therefore, the Yingxingzhai TTG gneisses most likely represent the upper crustal equivalent of the Hengshan TTG gneisses (Kröner et al. 2005a,b). This conclusion is consistent with the available geochemical data, which indicate that both the Hengshan and Yixingzhai TTG gneisses belong to calc-alkaline suites emplaced in a magmatic arc environment (Fig. 4; Kröner et al. $2005 a, b)$.

\section{4.e. 2350-2050 Ma: intermittent emplacement of Palaeoproterozoic granitoids}

Palaeoproterozoic granitoids in the Hengshan-WutaiFuping belt have not been recognized until recently, following acquisition of new SHRIMP and evaporation zircon ages which indicate the widespread presence of Palaeoproterozoic granitoids in the belt. In the Wutai Complex, Palaeoproterozoic granitoids are represented by the Dawaliang pluton and the pink phase of the Wangiiahui pluton, of which the former yielded a SHRIMP zircon age of $2176 \pm 12 \mathrm{Ma}$, whereas three samples of the latter were dated at $2117 \pm 17$,
$2116 \pm 16$ and $2084 \pm 20 \mathrm{Ma}$, respectively (Wilde et al. 2005). In the Fuping Complex, the Palaeoproterozoic granitoids are represented by the Nanying granitic gneisses which widely occur in the Fuping TTG gneisses (Fig. 4). SHRIMP data show that the Nanying gneissic granites were emplaced in the period 2077-2024 Ma (Table 1; Guan et al. 2002; Zhao et al. $2002 b$ ), slightly later than the Dawaliang granites. In the Hengshan Complex, similar-aged gneissic granites have been identified by Kröner et al. $(2005 a, b)$ using the SHRIMP and single-grain evaporation techniques (Table 1). They recognized three phases of Palaeoproterozoic gneissic granitoids that were emplaced at c. $2360 \mathrm{Ma}, 2330 \mathrm{Ma}$ and $2250 \mathrm{Ma}$, respectively (Table 1). It is particularly important to note that these Palaeoproterozoic granitoids in the Hengshan and Fuping complexes contain the same deformational features as the older Hengshan and Fuping granitoid gneisses and thus unambiguously demonstrate that the main deformational event is not Archaean but Palaeoproterozoic in age.

\section{4.f. 2150-1915 Ma: emplacement of pre-tectonic mafic dykes}

Pre-tectonic gabbro and diabase dykes are represented by numerous boudins and lensoid layers of mafic granulites or amphibolites in the Hengshan, Wutai and Fuping complexes. In some places, and in particular in low-strain zones such as those south of the Zhujiafang shear zone (Fig. 4), primary igneous fabrics (e.g. ophitic or subophitic texture) are well preserved in the amphibolite to granulite facies dykes (O'Brien, Walte \& Li, 2005; Kröner et al. 2005a, 2006). In most cases, and especially in high-strain zones such as the areas north of the Zhujiafang shear zone (Fig. 4), the pre-tectonic gabbroic/diabase dykes occur as highpressure granulites or retrograded eclogites, and strong ductile deformation has later rotated these dykes into parallelism with the layering in the enclosing gneisses and caused boudinage (Kröner et al. 2005a, 2006). In all of these cases the amphibolitic or granulitic layers and boudins are part of original gabbroic/diabase dykes that indicate an extensional event. In the Hengshan Complex, igneous zircons from two pre-tectonic mafic dykes (high-pressure granulites) yielded SHRIMP ${ }^{207} \mathrm{~Pb} /{ }^{206} \mathrm{~Pb}$ ages of $1915 \pm 4 \mathrm{Ma}$ and $1914 \pm 2 \mathrm{Ma}$ (Kröner et al. 2006), indicating that emplacement of the dykes occurred shortly before a major collisional (tectono-thermal) event at c. $1850 \mathrm{Ma}$ (see Section 4.h). The deformed and metamorphosed mafic dykes are also found in the Wutai Complex, and one amphibolite facies mafic dyke yielded a SHRIMP ${ }^{207} \mathrm{~Pb} /{ }^{206} \mathrm{~Pb}$ age of $2147 \pm 5 \mathrm{Ma}$ (P. Peng, unpub. Ph.D. thesis, Chinese Acad. Sciences, Beijing, 2005; Table 1). These mafic dykes were most probably emplaced in back-arc or intra-arc basin environments. 
4.g. 2100-1860 Ma: deposition of the late Palaeoproterozoic Hutuo Group and Wanzi supracrustal assemblage

The Hutuo Group is considered to be the youngest unit in the Hengshan-Wutai-Fuping belt and unconformably overlies both the Wutai and Fuping complexes (Tian, 1991). However, at several localities, the Hutuo rocks are tectonically interleaved with the Wutai 'Group', and at some localities the Hutuo Group shows the same deformational and metamorphic patterns as the Upper Wutai 'Subgroup'. This implies that deposition of the Hutuo Group rocks places a maximum age on the timing of the major deformational event in the Hengshan-Wutai-Fuping belt. A felsic tuffaceous rock collected from a metamorphosed sequence of volcanic rocks and sediments of the Hutuo Group contains two zircon populations with SHRIMP ${ }^{207} \mathrm{~Pb} /{ }^{206} \mathrm{~Pb}$ ages of $2180 \pm 5$ and $2087 \pm 9 \mathrm{Ma}$, respectively (Wilde et al. 2004b). The older age is within error of the age of the Dawaliang granite in the Wutai Complex and is considered to be derived from a similar crustal magmatic source. The younger age is within the error of reported ages for the Nanying granitic gneisses in the adjacent Fuping Complex and is interpreted to reflect the timing of Hutuo volcanism. The age of $2087 \pm 9 \mathrm{Ma}$ also means that the Hutuo rocks must have been deformed and metamorphosed after this time, which further supports the conclusion, obtained from other recent studies (Kröner et al. 2005a, 2006), that the main tectonism in the Hengshan-WutaiFuping belt occurred in the Palaeoproterozoic era and not in the late Archaean era as previously considered (Tian, 1991).

The Wanzi supracrustal assemblage in the Fuping Complex was previously considered to have been deposited between 2800 and $2560 \mathrm{Ma}$, constrained by conventional multigrain $\mathrm{U}-\mathrm{Pb}$ zircon ages (Liu et al. 1985). However, recent SHRIMP data do not support this conclusion. Zhao et al. (2002b) obtained SHRIMP ${ }^{207} \mathrm{~Pb} /{ }^{206} \mathrm{~Pb}$ zircon ages of $2502 \pm 7 \mathrm{Ma}$ and $2507 \pm 14 \mathrm{Ma}$ from two Al-rich gneiss samples of the Wanzi supracrustal assemblage, interpreted as the crystallization ages of the igneous protolith. These authors also obtained a concordant zircon age of $2109 \pm 5$ Ma from one Al-rich gneiss sample, which suggests that the protoliths of the Wanzi assemblage must have been deposited after c. 2109 Ma. This conclusion is supported by Guan et al. (2002), who obtained a SHRIMP zircon age of $2097 \pm 46 \mathrm{Ma}$ from a fine-grained paragneiss of the Wanzi assemblage. Most recently, Xia et al. (2006c) applied the LA-ICPMS technique to date the Wanzi supracrustal rocks and obtained three upper concordia intercept ages of $2099 \pm 22 \mathrm{Ma}, 2110 \pm 30 \mathrm{Ma}$ and $2112 \pm 23 \mathrm{Ma}$ for igneous zircon cores separated from a sillimanite paragneiss (Table 1). They also obtained metamorphic ages of 1863-1822 Ma for zircon rims that formed due to overgrowth or recrystallization. These data establish that (1) deposition of the Wanzi supracrustal assemblage occurred at some time between 2100 and $1860 \mathrm{Ma}$, and (2) that sedimentation of the Wanzi assemblage in the Fuping Complex was largely coeval with volcanism in the Hutuo Group of the Wutai Complex.

\section{4.h. 1880-1820 Ma: Late Palaeoproterozoic continent-continent collision}

In the Chinese literature, the high-grade Hengshan and Fuping complexes and the low-grade Wutai Complex have long been considered to be the products of two different tectono-metamorphic events, called the Fuping (c. $2.5 \mathrm{Ga}$ ) and Wutai (2.4-2.3 Ga) 'movements', respectively (Bai, 1986; Wu et al. 1989; Tian, 1991; Bai, Wang \& Guo, 1992; Bai \& Dai, 1998). This conclusion was based on a few 'unconformities', conventional multigrain zircon geochronology, and the common misconception that high-grade metamorphic rocks were older than low-grade ones. However, new geochronological data do not verify either the Fuping or Wutai 'movement'. In the Hengshan and Fuping complexes, SHRIMP U-Pb zircon studies combined with cathodoluminescence imaging and $\mathrm{U}_{-}$ Th chemistry confirm the existence of metamorphic zircons in most lithologies (Guan et al. 2002; Zhao et al. 2002b; Kröner et al. 2005a,b, 2006). These zircons occur either as overgrowth rims surrounding older magmatic cores or as single, metamorphic grains, and are structureless, highly luminescent, multifaceted and very low in Th and $U$ contents. These features make them distinctly different from the igneous zircons that are generally characterized by oscillatory zoning and comparatively high Th and U contents. Most metamorphic zircons from both Neoarchaean and Palaeoproterozoic rocks in the Fuping and Hengshan complexes yielded similar concordant ages in the range 1880 to $1820 \mathrm{Ma}$ (Table 1), which are $700 \mathrm{Ma}$ to $150 \mathrm{Ma}$ younger than their igneous zircon cores. In the low- to medium-grade Wutai Complex, although metamorphic zircons have not yet been observed, a garnet-amphibole-whole rock $\mathrm{Sm}-\mathrm{Nd}$ isochron age of $1851 \pm 9 \mathrm{Ma}$ was obtained for a garnet amphibolite collected from the Lower Wutai 'Subgroup' (Table 1; Wang et al. 2001). Most recently, Liu et al. (2004b, 2006) applied the electron probe microanalysis (EPMA) monazite dating technique to determination of metamorphic ages of the Wutai Complex. They obtained $\mathrm{ThO}_{2} * / \mathrm{PbO}$ ages of $1833 \pm 8,1822 \pm 14$ and $1846 \pm 64$ Ma for three monazite grains separated from a garnet-kyanite mica schist from the lower part (called the Jingangku Formation) of the 'Shizui Subgroup' (see Fig. 4). These data are consistent with the above mineral $\mathrm{Sm}-\mathrm{Nd}$ isochron age of $1851 \pm 9 \mathrm{Ma}$. Taken together, these results indicate that the main regional metamorphism of the Hengshan, Wutai and 


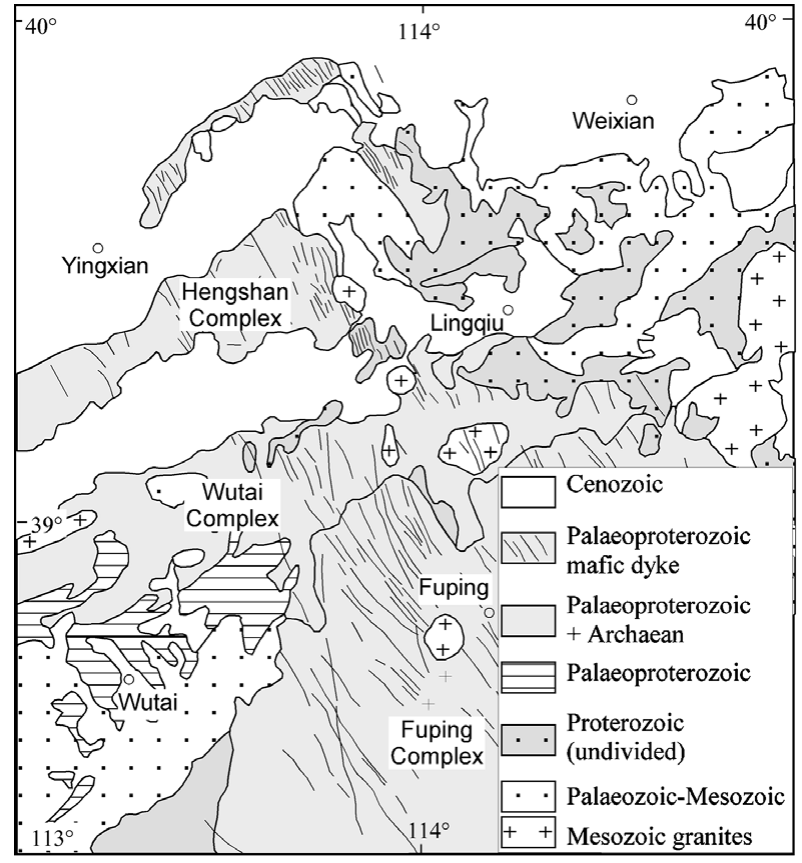

Figure 7. Spatial distribution of post-tectonic mafic dykes in the Hengshan-Wutai-Fuping belt (after Zhao et al. 2001b).

Fuping complexes occurred during the $1880-1820 \mathrm{Ma}$ 'Lüliang Movement'. Based on these metamorphic ages, we conclude that this major tectonothermal event at c. $1.85 \mathrm{Ga}$ is related to collision between the Eastern and Western blocks to form the North China Craton.

\section{4.i. 1780-1750 Ma: emplacement of post-orogenic mafic dyke swarm}

Late Palaeoproterozoic mafic dykes are widespread in the Hengshan-Wutai-Fuping belt, with a predominant NW-SE to NNW-SSE trend and, on a regional scale, constitute a dyke swarm (Fig. 7). Generally, they dip steeply, cutting both the Archaean and Palaeoproterozoic basement rocks and are covered by Mesoproterozoic and younger strata. Individual dykes range in width from 10 to $50 \mathrm{~m}$ to a maximum of about $100 \mathrm{~m}$, and in length from 10 to $40 \mathrm{~km}$, to a maximum of about $100 \mathrm{~km}$. Most dykes are unmetamorphosed and undeformed, with chilled contacts. However, a few dykes were metamorphosed to upper amphibolite facies, indicated by garnet and amphibole reaction rims surrounding igneous plagioclase and clinopyroxene grains. Using the single-grain dissolution technique, Halls et al. (2000) obtained an upper concordia intercept zircon age of $1769.1 \pm 2.5 \mathrm{Ma}$ for an unmetamorphosed diabase dyke in the Hengshan Complex. Applying the same technique, Peng (P. Peng, unpub. Ph.D. thesis, Chinese Acad. Sciences, Beijing, 2005) obtained a similar upper intercept age of $1754 \pm 71 \mathrm{Ma}$ for an undeformed and unmetamorphosed mafic dyke in the Wutai Complex. The post-tectonic mafic dykes in the Fuping Complex have not been well dated, but Wang et al. (2004a) obtained whole-rock ${ }^{40} \mathrm{Ar}-$ ${ }^{39} \mathrm{Ar}$ ages of $1765.3 \pm 1.1 \mathrm{Ma}, 1774.7 \pm 0.7 \mathrm{Ma}$ and $1780.7 \pm 0.5 \mathrm{Ma}$ for mafic dykes in the Zanhuang Complex, which is $50 \mathrm{~km}$ south of the Fuping Complex (Fig. 3), and the two together constitute the Taihang Mountain range. All of these data suggest that emplacement of undeformed and largely unmetamorphosed mafic dykes in the Hengshan-Wutai-Fuping belt occurred in the period 1780-1750 Ma. Controversy has surrounded the origin of these mafic dykes, with one school of thought believing that they were the products of a post-collisional extensional event (Zhao et al. $2001 b$ ), whereas others argued that they were produced from a mantle plume event that led to break-up of the hypothetical Palaeo-Mesoproterozoic supercontinent Columbia (Zhai, Bian \& Zhao, 2000; P. Peng, unpub. Ph.D. thesis, Chinese Acad. Sciences, Beijing, 2005).

\section{Discussion}

Major lithotectonic assemblages and geological events in the Hengshan-Wutai-Fuping belt, as listed in Table 1 , enable us to place rigorous constraints on a number of important geological issues regarding the Neoarchaean to Palaeoproterozoic evolution of this belt and the Trans-North China Orogen.

\section{5.a. Relationships between the high-grade Hengshan and Fuping gneiss complexes and low-grade Wutai granite-greenstone terrane}

Two contrasting tectonic models have previously been proposed for the evolution of the Hengshan, Fuping and Wutai complexes. One suggests that the first two developed as a single continental block that underwent Neoarchaean rifting associated with formation of the Wutai greenstones and closed upon itself in the Palaeoproterozoic era (Tian, 1991; Yuan \& Zhang, 1993), while the other model proposes that the entire terrane is a Neoarchaean continent-arc-continent collision system, in which the Hengshan and Fuping complexes represent two exotic Archaean microcontinental blocks, and the Wutai granite-greenstone represents an intervening island arc (Bai, 1986; Li et al. 1990; Bai, Wang \& Guo, 1992; Wang et al. 1996; Polat et al. 2005). Although these two models propose different tectonic settings and processes for the evolution of these complexes, they both assume that the Hengshan and Fuping gneisses are a crystalline basement to the Wutai granite-greenstone terrane. However, this assumption is not supported by the isotopic age data summarized above. As shown in Table 1, the Hengshan, Wutai and Fuping complexes are not remarkably different in age and all formed during Neoarchaean to Palaeoproterozoic times, although the Wutai granitoids are slightly older than major lithologies of the Hengshan and Fuping complexes. These three complexes 
seem to have experienced a common Neoarchaean to Palaeoproterozoic evolution, with the low- to mediumgrade Wutai Complex representing an upper crustal calc-alkaline volcano-plutonic assemblage, probably generated above a subduction zone in a magmatic arc setting, whereas the Hengshan and Fuping complexes may be the lower crustal root zone of the arc. Thus, the tectonic evolution of the Hengshan-Wutai-Fuping belt may not be related to local interaction of the three complexes, either through closure of a Wutai rift (Tian, 1991; Yuan \& Zhang, 1993) or collision between a Wutai arc and the putative Hengshan and Fuping microcontinental blocks (Bai, 1986; Bai, Wang \& Guo, 1992; Li et al. 1990; Wang et al. 1996; Polat et al. 2005). Instead, we suggest that the three complexes represent elements of a single Neoarchaean to Palaeoproterozoic, long-lived magmatic arc that was subsequently incorporated into the Trans-North China Orogen during collision between the Eastern and Western blocks at around $1.85 \mathrm{Ga}$ (Zhao et al. 2000b, 2001b, 2002a; Wilde, Zhao \& Sun, 2002).

\section{5.b. Nature of the Hengshan-Wutai-Fuping magmatic arc}

Available geochemical data for rocks of the Hengshan, Fuping and Wutai complexes support an arc derivation (Geng \& Wu, 1990; Li et al. 1990; Bai, Wang \& Guo, 1992; Sun, Armstrong \& Lambert, 1992; Wang et al. 1996; Liu et al. 2002b, 2004a,b, 2005; Wang et al. 2004b; Kröner et al. 2005a,b), with minor ultramafic to mafic rocks in the Lower Wutai that have been interpreted as remnants of ancient oceanic crust (Bai, Wang \& Guo, 1992; Wang et al. 1996). Bai, Wang \& Guo (1992) showed that the majority of amphibolites from the Lower Wutai and greenschists from the Middle Wutai have an affinity to calc-alkaline basalts or arc tholeiites (Fig. 5b). Wang et al. (2004b) also showed that the majority of amphibolites and greenschists in the Wutai 'Group' were derived from island arc-type basalts, though minor amounts have an affinity to MORB-type and back-arc basin basalts (Fig. 5c). Based on major and trace element features and Nd isotopes, Kröner et al. $(2005 a, b)$ showed that the Hengshan and Fuping TTG gneisses are characterized by a wide range in $\mathrm{SiO}_{2}$ content, high $\mathrm{Na}_{2} \mathrm{O}, \mathrm{Ba}, \mathrm{Sr}$ and low $\mathrm{Y}$ and HREE, and their selective enrichment in LIL elements and depletion in $\mathrm{Nb}$, Ta and $\mathrm{Ti}$ can be derived from magmatic precursors with a strong mantle signature, modified by a subduction component and variable contributions from older crust. A particularly diagnostic discriminant for the tectonic setting is the $\mathrm{Rb}-\mathrm{Hf}-\mathrm{Ta}$ triangular plot for felsic to intermediate magmatic rocks, in which arc-derived assemblages occupy a field distinct from those generated in withinplate and ocean floor settings (Harris, Pearce \& Tindle, 1986), though this diagram is useful only when $\mathrm{Rb}$ has not been mobilized. Figure 5a shows such a plot for the Hengshan TTG gneisses (Kröner et al. 2005a,b;
Liu et al. 2004a), Wutai volcanic and granitoid rocks (Wang et al. 2004b; Liu et al. 2004b) and Fuping TTG gneisses (Liu et al. 2004a), clearly supporting their arc derivation. However, controversy still remains as to the nature of this magmatic arc. In most previous models, the Wutai Complex was considered to have formed in a Mariana-type arc system that was initiated by intraoceanic subduction (Li et al. 1990; Bai, Wang \& Guo, 1992; Wang et al. 1996). Based on the presence of minor MORB-type basalts and adakite-featured felsic volcanic rocks in the 'Wutai Group', Wang et al. (2004b) also preferred an intra-oceanic subduction process for the formation of the 2.6-2.5 Ga Wutai granite-greenstone terrane. However, the Marianatype arc model is not supported by recent age data, which reveal the widespread existence of remnants of 2.66-2.82 Ga old continental crust in the Hengshan, Wutai and Fuping complexes. This is indicated by c. $2.7 \mathrm{Ga}$ medium-grained grey biotite or hornblende gneisses that occur interlayered with the younger Hengshan and Fuping TTG gneisses (Guan et al. 2002; Kröner et al. 2005b), 2.67-2.76 Ga xenocrystic zircons from the Wutai and Longquanguan granitoid gneisses (Wilde, Cawood \& Wang, 1997; Wilde et al. 2004a; Wilde, 2002), and 2.68-2.82 Ga detrital zircons from the Wanzi supracrustal rocks (Zhao et al. 2002b). More importantly, Wilde et al. (2004a) found a group of $2679 \pm 16 \mathrm{Ma}$ old xenocrystic zircons in a $2529 \pm 10 \mathrm{Ma}$ meta-andesite from the Zhuangwang 'Formation' that is considered part of the Shizui 'Subgroup'. These data suggest that the HengshanWutai-Fuping belt was not an intra-oceanic arc but seems to have developed on a continental margin. The available data do not allow us to determine further whether it was a Japan-type island arc or an Andeantype continental margin magmatic arc. Although minor MORB-type basalts have been reported from the Wutai Complex (Wang et al. 2004b), it remains uncertain whether these erupted in a back-arc basin or a marginal basin environment.

\section{5.c. Trans-North China Orogen: a long-lived (c. $700 \mathrm{Ma})$ accretionary magmatic arc?}

As shown in Table 1, much of the juvenile crust of the Hengshan-Wutai-Fuping belt was accreted in the period 2560-2480 Ma, though a number of magmatic accretionary events occurred during Palaeoproterozoic times. However, more than $90 \%$ of juvenile crust in other complexes (e.g. the Lüliang, Zhongtiao, Northern Hebei complexes; Fig. 3) in the Trans-North China Orogen was added in the period 2300-1900 Ma (Sun et al. 1993; Sun, 1997; Yu, Wang \& Wang, 1997; Geng et al. 2000; Tian et al. 2005). This suggests that the Trans-North China Orogen records magmatictectonic evolution lasting for nearly $650 \mathrm{Ma}$. This is in contrast to most Phanerozoic collision belts where pre-orogenic sedimentation and magmatism are 
followed, within tens of millions of years, by orogenic deformation and metamorphism. The long-lived nature of the Trans-North China Orogen has been regarded as a major argument against the $1.85 \mathrm{Ga}$ collision model, since few convergent continental-margin arcs in the world sat undisturbed for $c$. 700 Ma before being deformed and metamorphosed during accretionary and collisional events (Kusky \& Li, 2003). However, such long-lived arcs have been documented or inferred in many other orogenic belts (Rivers \& Corrigan, 2000), as summarized below. Kröner, Klemd \& Zhao (2006) discussed the evolution of two such long-lived continental collisional belts, the Central Zone of the Limpopo belt in southern Africa and the Hengshan Complex of the Trans-North China Orogen, both of which consist predominantly of Archaean granitoid gneisses and minor shallow-water sediments with minor additions of early Palaeoproterozoic granites, and which were subjected to intense deformation and metamorphism in the late Palaeoproterozoic era, 500 $700 \mathrm{Ma}$ after their generation in arc-related settings. As pointed out by Kröner, Klemd \& Zhao (2006), in both cases continental collision has been inferred, but there is no record of pre-collisional continental margin evolution and ocean closure; instead both terranes show evidence of having been generated in magmatic arc environments. These authors suggested that these terranes were rifted off from unknown Archaean active continental margins during Palaeoproterozoic times, similar to present-day Japan, and were then displaced before becoming attached, through terrane collision, to different Archaean blocks with which they share no common history.

Similar Palaeo-Mesoproterozoic long-lived magmatic arcs have also been found in southeastern Laurentia, southern Baltica, central Australia and western Amazonia (Karlstrom et al. 2001; Bingen et al. 2002; Brewer et al. 2002). In southeastern Laurentia and southern Baltica, a 1.8 to 1.3 Ga magmatic arc zone extends from Arizona through Colorado, Michigan, southern Greenland, Scotland, Sweden and Finland to western Russia, bordering the present southern margin of North America, Greenland and Baltica (Gower, Ryan \& Rivers, 1990; Karlstrom et al. 2001). Petrological and geochemical studies indicate that this large magmatic arc zone includes dominantly juvenile volcanogenic sequences and granitoid suites resembling those of present-day island arcs and active continental margins (Nelson \& DePaolo, 1985; Bennet \& DePaolo, 1987) and may represent subduction-related episodic outgrowth along the continental margin of a Palaeo-Mesoproterozoic supercontinent (Karlstrom et al. 2001; Zhao et al. 2002a, 2004).

The Central Asian Orogenic Belt and the Kunlun Orogens are good examples of Phanerozoic long-lived magmatic arcs (Enkin et al. 1992; Sengör, Natalin \& Burtman, 1993; Heubeck, 2001; Windley et al. 2002, 2007; Xiao et al. 2003, 2004, 2005; Jahn et al. 2004).
The Central Asian Orogenic Belt, also called the Altaids (Sengör, Natalin \& Burtman, 1993), is situated between the Siberian and Sino-Korean-Tarim cratons, and encompasses an immense area $(c .1000 \mathrm{~km}$ wide and c. $7000 \mathrm{~km}$ long) from the Urals in the west, through Kazakhstan, NW China, Mongolia, NE China to the Okhotsk Sea in the Russian Far East (Jahn et al. 2004). Available data show that the Central Asian Orogenic Belt was the world's largest site of Phanerozoic juvenile crust that developed from early Palaeozoic to early Mesozoic times (Sengör, Natalin \& Burtman, 1993; Windley et al. 2002, 2007; Xiao et al. 2003, 2004; Jahn et al. 2004). A present-day example of long-lived magmatic arcs is the Andes, where the Pacific plate has been subducting under the west coast of South America for c. 500 million years since the Cambrian (Howell, 1995; Dalziel, 1997; Rivers \& Corrigan, 2000). The Japanese arc has also existed since the Early Permian (Howell, 1995). These examples demonstrate that such long-lived arc magmatism is not unique, though the mechanism maintaining such long-lived arc magmatism remains enigmatic. Rivers \& Corrigan (2000) argued that longlived magmatic arcs are generally large geological features and may have faced a major open ocean, like the present-day Andes and Japanese arcs. This is consistent with our present model for the TransNorth China Orogen inferring a major ocean between the Eastern and Western blocks from before $2.55 \mathrm{Ga}$ until its final closure at $1.85 \mathrm{Ga}$.

\section{5.d. Palaeoproterozoic magmatic events and their regional significance}

Most of the crust in the Hengshan-Wutai-Fuping belt was formed during Neoarchaean times, including the 2560-2520 Ma Wutai and Longquanguan granitoids, 2530-2515 Ma Wutai greenstone-type volcanics, and 2520-2480 Ma Hengshan, Yixingzhai and Fuping grey TTG gneisses (Table 1). These lithologies occupy more than $70 \%$ of the total exposure of the HengshanWutai-Fuping belt (Fig. 4). As discussed above, all these rocks have affinities to modern magmatic arcs and are considered to have formed in a long-lived Japantype or Andes-type arc.

In the Chinese literature, all lithologies in the Hengshan-Wutai-Fuping belt, except for the Hutuo Group, were considered to be Archaean in age (Bai, 1986; Tian, 1991; Bai, Wang \& Guo, 1992). However, the zircon ages summarized above and in Table 1 reveal the widespread presence of Palaeoproterozoic granitoids in the Hengshan, Wutai and Fuping complexes. The presence of these ductilely deformed Palaeoproterozoic granitoid gneisses in the Hengshan and Fuping complexes indicates that the major deformation in these complexes is not Archaean but Palaeoproterozoic in age. This is inconsistent with the models of Kusky \& Li (2003) and Polat et al. (2005), 
which suggested that the collisional event leading to major deformation in these complexes occurred at $c$. $2.5 \mathrm{Ga}$.

Another important Palaeoproterozoic igneous event revealed by isotopic studies is the emplacement of pre-tectonic mafic dykes which were boudinaged and transformed to high- to medium-pressure mafic granulites in the Hengshan and Fuping complexes and garnet-bearing amphibolites in the Wutai Complex. The precursors of these mafic granulites have been considered to be Archaean in age because Tian et al. (1992) obtained a controversial Sm-Nd whole-rock isochron age of $2818 \pm 86$ Ma for these rocks from the Hengshan Complex. However, the new ages for igneous zircons from two metamorphosed mafic dykes in the Hengshan Complex (Table 1) undoubtedly indicate that they were emplaced in the Palaeoproterozoic era.

\section{A tectonic scenario for the evolution of the Hengshan-Wutai-Fuping belt}

On the basis of lithological, structural, metamorphic, geochemical and geochronological data summarized in this paper, we propose the following scenario for the evolution of the Hengshan-Wutai-Fuping belt, as shown in Figure 8.

(1) During Neoarchaean times, the HengshanWutai-Fuping region was part of an Andeantype arc along the western margin of the Eastern Block, which was separated from the Western Block by a major ocean, with subduction of the oceanic lithosphere beneath the western margin of the Eastern Block (Fig. 8a). At 2560 $2520 \mathrm{Ma}$, the release of water from the downgoing slab caused partial melting of the lower crust in the overriding plate, producing large volumes of granitoid magma that were emplaced into the upper levels of the crust to form the Wutai and Longquanguan granitoid rocks (Fig. 8a).

(2) At 2530-2515 Ma, subduction of oceanic lithosphere and further release of fluids caused partial melting in the overriding mantle wedge, leading to underplating of mafic magmas in the lower crust and widespread mafic to felsic volcanism, forming part of the Wutai greenstone assemblage (Fig. 8b). Extension driven by widespread mafic to felsic volcanism led to the development of a back-arc basin or marginal sea which divided the region into the Hengshan-Wutai island arc (Japan-type) and the Fuping relict arc. Minor MORB-type basalts and ultramafic rocks from the Wutai greenstone assemblage were formed in the back-arc basin or marginal sea (Fig. 8b).

(3) At 2520-2480 Ma, subduction beneath the Hengshan-Wutai island arc triggered partial melting of the lower crust to form large amounts of TTG magmas that crystallized into the Hengshan and Yixingzhai TTG suites (Fig. 8c). Meanwhile, eastward-directed subduction of oceanic lithosphere of the marginal sea led to reactivation of the Fuping relict arc, where the Fuping TTG suite was emplaced (Fig. 8c).

(4) In the Palaeoproterozoic era (2360-2000 Ma), several phases of granitoid magmatism occurred in both the Hengshan-Wutai island arc and the Fuping reactivated arc (Fig. 8d), represented by the $2360 \mathrm{Ma}$, c. $2250 \mathrm{Ma}$ and 2000-2100 Ma granitoid gneisses in the Hengshan Complex (Kröner et al. 2005a,b), c. 2100 Ma Wangjiahui and Dawaliang granites in the Wutai Complex (Wilde et al. 2005), and 2100-2000 Ma Nanying granitoid gneisses in the Fuping Complex (Zhao et al. 2002b; Guan et al. 2002; Liu et al. 2002b, 2005).

(5) At c. $1920 \mathrm{Ma}$, the Hengshan-Wutai island arc underwent an extensional event, possibly due to subduction of a mid-ocean ridge, leading to the emplacement of a major mafic dyke swarm (Fig. 8e). These dykes were subsequently metamorphosed to medium- to high-pressure granulites or retrograded eclogites in the Hengshan Complex and some amphibolites in the Wutai Complex.

(6) At 1880-1820 Ma, closure of the ocean between the Eastern and Western blocks led to continentarc-continent collision (Fig. 8f). This collision induced large-scale thrusting, tectonic imbrication and isoclinal folding (Fig. 8f) and transported part of the Hengshan and Fuping lithologies into deep crustal levels where granulite and eclogite facies metamorphism occurred, whereas the Wutai Complex underwent greenschistto lower amphibolite-facies metamorphism at higher crustal levels. Following peak metamorphism, the thickened crust underwent exhumation and near-isothermal decompression, forming widespread symplectic textures in the rocks. Further exhumation and inflow of fluids along ductile extensional shear zones led to cooling and retrogression, exemplified by widespread migmatization and in situ melting in the granitoid gneisses.

(7) The last magmatic event in the HengshanWutai-Fuping belt was the emplacement of a 1780-1750 Ma mafic dyke swarm, probably as a result of orogenic collapse or post-orogenic extension.

Other metamorphic complexes in the Trans-North China Orogen document a Neoarchaean to Palaeoproterozoic history essentially similar to that of the Hengshan-Wutai-Fuping belt. For example, the Huai'an, Xuanhua, Chengde and Taihua complexes (Fig. 3) can be compared to the Hengshan or 


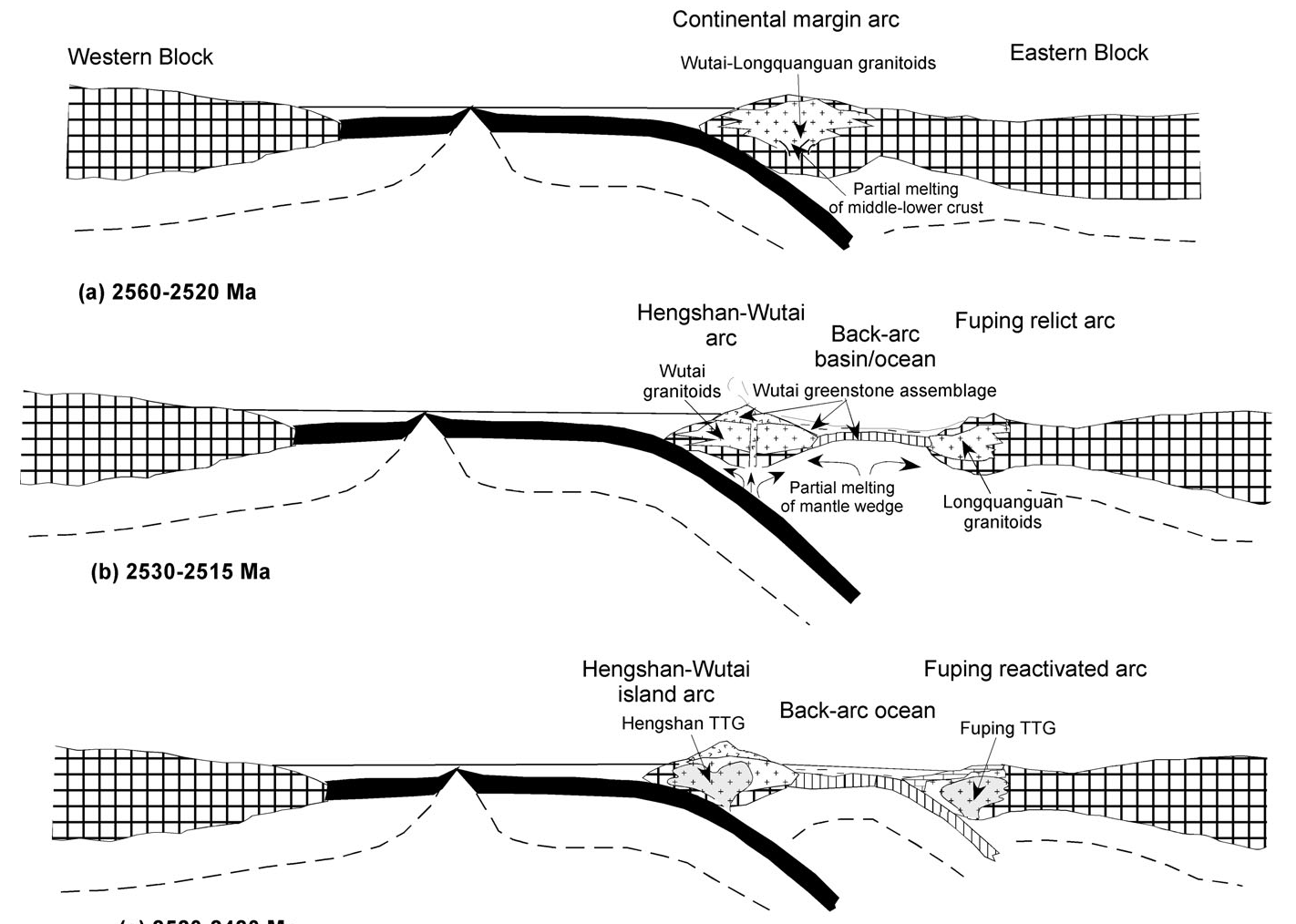

(c) 2520-2480 Ma

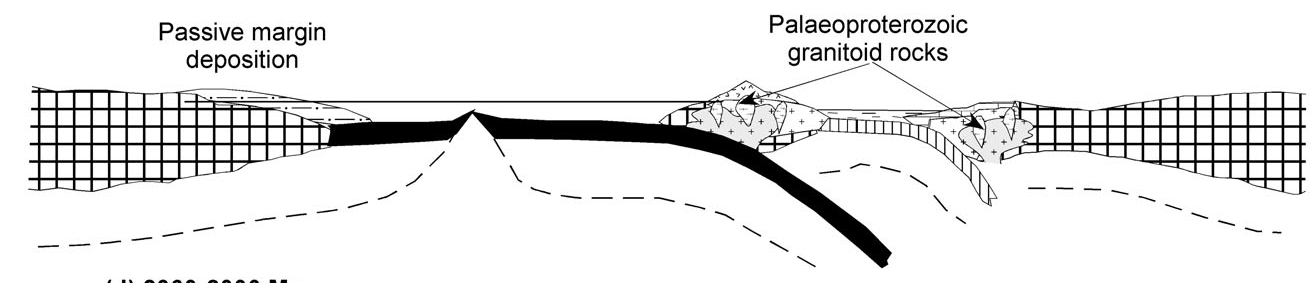

(d) $2360-2000 \mathrm{Ma}$

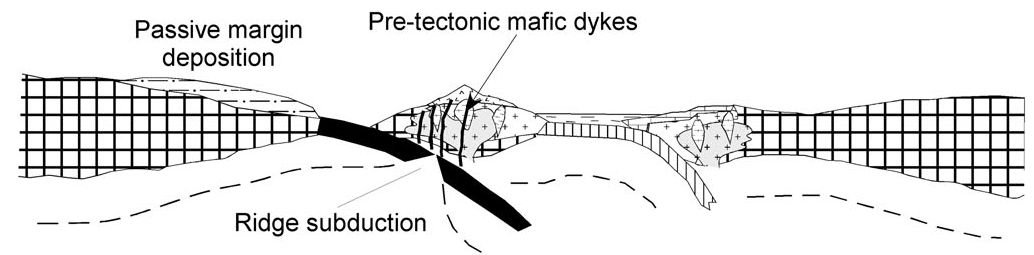

(e) $\sim 1920 \mathrm{Ma}$

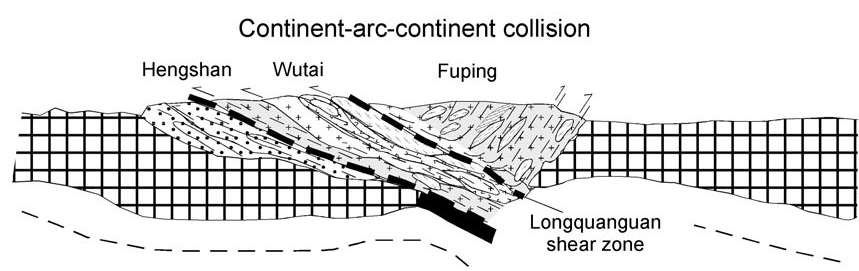

(f) 1880-1820 Ma Trans-North China Orogen

Figure 8. Schematic and speculative sections showing proposed geological evolution of the Hengshan-Wutai-Fuping belt and amalgamation of the North China Craton.

Fuping complexes, whereas the Zhongtiao, Dengfeng, Zanhuang and Northern Hebei complexes (Fig. 3) are similar to the Wutai Complex (Zhao et al. 2000a). Therefore, the magmatic, structural and metamorphic history of the Hengshan-Wutai-Fuping belt as summarized in this paper provides important insights for understanding the tectonic evolution of the Trans-North China Orogen. 
Acknowledgements. This research was financially supported by Hong Kong RGC grants (HKU7055/05P, 7048/04P, $7055 / 03 \mathrm{P}$ and 7048/03P), China NSFC grants (40429001, 40420120135 and 40472098) and a HKU Seed Funding for Basic Research (200411159122), all to G.Z. A.K. and S.W. acknowledge funding of the German Science Foundation (DFG, Grant KR 590/XX) and an Australia ARC project, respectively. We appreciate discussions with our colleagues Mingguo Zhai, Jinghui Guo, Changhua Wu, Peter Cawood, Jinghai Li, Timothy Kusky, Patrick O’Brien, Cees Passchier, and participants at the Beijing Penrose Conference in 2002. This is the Institute of Geosciences (TIGER) Publication No. 1. AK's authorship forms contribution no. 43 of the Geocycles Cluster of Mainz University, funded by the State of Rhineland-Palatinate, Germany.

\section{References}

BAI, J. 1986. The Precambrian crustal evolution of the Wutaishan area. In The Early Precambrian Geology of Wutaishan (ed. J. Bai), pp. 376-83. Tianjing: Tianjin Science and Technology Press.

BAI, J. \& DAI, F. Y. 1998. Archaean crust of China. In Precambrian Crust Evolution of China (eds X. Y. Ma \& J. Bai), pp. 15-86. Beijing: Springer-Geological Publishing House.

BAI, J., WANG, R. Z. \& GUO, J. J. 1992. The Major Geologic Events of Early Precambrian and Their Dating in Wutaishan Region. Beijing: Geological Publishing House (in Chinese), pp. 34-55.

Bennet, V. C. \& DePaOlO, D. J. 1987. Proterozoic crustal history of the western United States as determined by neodymium isotopic mapping. Geological Society of America Bulletin 99, 674-85.

Bingen, B., Mansfeld, J., Sigmond, E. M. O. \& Stein, H. 2002. Baltica-Laurentia link during the Mesoproterozoic: $1.27 \mathrm{Ga}$ development of continental basins in the Sveconorwegian Orogen, southern Norway. Canadian Journal of Earth Sciences 39, 1425-40.

Brewer, T. S., Ahall, K. I., Darbyshire, D. P. F. \& MENUGE, J. F. 2002. Geochemistry of late Mesoproterozoic volcanism in southwestern Scandinavia: implications for Sveconorwegian-Grenvillian plate tectonic models. Journal of the Geological Society, London 159, $129-44$.

Condie, K. C. 1994. Greenstones through time. In Archean Crustal Evolution (ed. K. C. Condie), pp. 85-120. Amsterdam: Elsevier.

CondiE, K. C. 1997. Sources of Proterozoic mafic dyke swarms: Constraints from $\mathrm{Th} / \mathrm{Ta}$ and $\mathrm{La} / \mathrm{Yb}$ ratios. Precambrian Research 81, 3-14.

Condie, K. C. 2005. High field strength element ratios in Archean basalts: a window to evolving sources of mantle plumes? Lithos 79, 491-504.

DALZIEL, I. W. D. 1997. Neoproterozoic-Paleozoic geography and tectonics: review, hypothesis, environmental speculation. Geological Society of America Bulletin 108, $16-42$.

DU, W. W., WANG, R. M. \& Wang, G. Y. 1999. Some clues to the presence of palaeo-ophiolites in the eastern part of the Dandong-Kuandian areas. Journal of Peking University (Natural Science) 35, 254-8.

Enkin, R. J., YANG, Z., Chen, Y. \& COURTILlot, V. 1992. Paleomagnetic constraints on the geodynamic history of the major blocks of China from Permian to the present. Journal of Geophysical Research 97B, 13953-89.
GE, W. C., ZhaO, G. C., Sun, D. Y., WU, F. Y. \& Lin, Q. 2003. Metamorphic $P-T$ path of the Southern Jilin complex: Implications for tectonic evolution of the Eastern block of the North China craton. International Geology Review 45, 1029-43.

Geng, Y. S., Wan, Y. S., Shen, Q. H., Li, H. M. \& ZHANG, R. X. 2000. Chronological framework of the Early Precambrian important events in the Lüliang Area, Shanxi Province. Acta Geologica Sinica (English edition) 74, 216-23.

GENG, Y. S. \& WU, J. S. 1990. Geochemistry and evolution of the early Precambrian mafic rocks in the WutaiTaihangshan area. Precambrian Geology 4, 167-74 (in Chinese).

Gower, C. F., Ryan, A. B. \& Rivers, T. 1990. MidProterozoic Laurentia-Baltica: An overview of its geological evolution and summary of the contributions by this volume. In Mid-Proterozoic Laurentia-Baltica (eds C. F. Gower, T. Rivers \& B. Ryan), pp. 120. Geological Association of Canada, Special Paper no. 38 .

Guan, H., Sun, M., Wilde, S. A., Zhou, X. H. \& Zhai, M. G. 2002. SHRIMP U-Pb zircon geochronology of the Fuping Complex: implications for formation and assembly of the North China craton. Precambrian Research 113, 1-18.

GuO, J. H., O’BRIEN, P. J. \& ZHAI, M. G. 2002. High-pressure granulites in the Sangan area, North China Craton: metamorphic evolution, $P-T$ paths and geotectonic significance. Journal of Metamorphic Geology 20, 74156.

GuO, J. H., ShI, X., BiAn, A. G., Xu, R. H., ZhaI, M. G. \& LI, Y. G. 1999. Pb isotopic compositions of feldspar and $\mathrm{U}-\mathrm{Pb}$ age of zircons from early Proterozoic granites in the Sanggan area, North China Craton: metamorphism, crustal melting and tectonothermal events. Acta Petrologica Sinica 15, 199-207.

Guo, J. H., Sun, M. \& ZHAI, M. G. 2005. Sm-Nd and SHRIMP U-Pb zircon geochronology of high-pressure granulites in the Sanggan area, North China Craton: Timing of Palaeoproterozoic continental collision. Journal of Asian Earth Sciences 24, 629-42.

GuO, J. H., Wang, S. S., SANG, H. Q. \& ZHAI, M. G. 2001. Ar40-Ar39 age spectra of garnet porphyroblast: Implications for metamorphic age of high-pressure granulite in the North China Craton. Acta Petrologica Sinica 17, 436-42.

GuO, J. H. \& ZHAI, M. G. 2001. Sm-Nd age dating of highpressure granulites and amphibolites from Sanggan area, North China Craton. Chinese Science Bulletin 46, 10611.

Halls, H. C., Li, J. H., Davis, D., Hou, G., Zhang, B. X. \& QIAN, X. L. 2000. A precisely dated Proterozoic palaeomagnetic pole from the North China craton, and its relevance to palaeocontinental reconstruction. Geophysical Journal International 143, 185-203.

HaO, D. F., Li, S. Z., ZhaO, G. C., Sun, M., HaN, Z. Z. \& ZHAO, G. T. 2004. Origin and its constraint to tectonic evolution of Palaeoproterozoic granitoids in the eastern Liaoning and Jilin province, North China. Acta Petrologica Sinica 20, 1409-16.

Harris, N. B. W., Pearce, J. A. \& Tindle, A. G. 1986. Geochemical characteristics of collision-zone magmatism. In Collision tectonics (eds M. P. Coward \& A. C. Ries), pp. 67-81. Geological Society of London, Special Publication no. 19. 
Heubeck, C. 2001. Assembly of central Asia during the middle and late Paleozoic. In Paleozoic and Mesozoic tectonic evolution of central and eastern Asia (eds M. S. Hendrix \& G. A. Davis), pp. 1-22. Geological Society of America, Memoir no. 192.

Howell, D. G. 1995. Principles of terrane analysis: new applications for global tectonics. 2nd ed. London: Chapman and Hall.

Jahn, B. M., Auvray, B., Cornichet, J., Bai, Y. D., Shen, Q. H. \& LIU, D. Y. 1987. 3.5 Ga old amphibolites from eastern Hebei Province, China: field occurrence, petrography, Sm-Nd isochron age and REE geochemistry. Precambrian Research 34, 311-46.

JAHN, B. M., AUVRAY, B., SHEN, Q. H., LIU, D. Y., ZHANG, Z. Q., DONG, Y. J., YE, X. J., ZHANG, Q. Z., CORNICHET, J. \& MACE, J. 1988. Archaean crustal evolution in China: the Taishan Complex and evidence for Juvenile crustal addition from long-term depleted mantle. Precambrian Research 38, 381-403.

JAHN, B. M. \& ERNST, W. G. 1990. Neoarchaean Sm-Nd Isochron Age for Mafic-Ultramafic Supracrustal Amphibolites from the Northeastern Sino-Korean Craton, China. Precambrian Research 46, 295-306.

JAHN, B. M., WindLEY, B., NATAL'IN, B. \& DOBRETSOV, N. 2004. Phanerozoic continental growth in central Asia Preface. Journal of Asian Earth Sciences 23, 599-603.

JAHN, B. M. \& ZHANG, Z. Q. 1984. Archaean granulite gneisses from eastern Hebei Province, China: rare earth geochemistry and tectonic implications. Contributions to Mineralogy and Petrology 85, 224-43.

Karlstrom, K. E., Harlan, S. S., ÅHÄLl, K. I., WilliamS, M. L., Mclelland, J. \& Geissman, J. W. 2001. Long-lived (1.8-1.0 Ga) convergent orogen in southern Laurentia, its extensions to Australia and Baltica, and implications for refining Rodinia. Precambrian Research 111, 5-30.

KRÖNER, A. 2002. Zircon ages of the Hengshan Complex. In A Neoarchaean to Palaeoproterozoic Lower to Upper Crustal Section in the Hengshan-Wutaishan Area of North China (eds A. Kröner, G. C. Zhao, S. A. Wilde, M. G. Zhai, C. W. Passchier, M. Sun, J. H. Guo, P. J. O'Brien \& N. Walte), pp. 28-32. Guidebook for Penrose Conference Field Trip, September 2002. Beijing: Chinese Academy of Sciences.

Kröner, A., Compston, W., Zhang, G. W., Guo, A. L. \& TODT, W. 1988. Ages and tectonic setting of Neoarchaean greenstone-gneiss terrain in Henan Province, China, as revealed by single-grain zircon dating. Geology 16, 211-15.

Kröner, A., CUI, W. Y., WANG, W. Y., WANG, C. Q. \& Nemchin, A. A. 1998. Single zircon ages from high-grade rocks of the Jianping Complex, Liaoning Province, NE China. Journal of Asian Earth Sciences 16, 519-32.

KRÖNer, A., KlemD, R. \& ZHAO, G. C. 2006. Rates of orogenic processes in two unusual Palaeoproterozoic high-grade terrains of southern Africa and northern China, EUG, Vienna. Geophysical Research Abstract 8, 2796-7.

KRÖNER, A., Wilde, S. A., LI, J. H. \& WANG, K. Y. 2005a. Age and evolution of a Neoarchaean to early Palaeozoic upper to lower crustal section in the Wutaishan/Hengshan/Fuping terrain of northern China. Journal of Asian Earth Sciences 24, 577-95.

Kröner, A., Wilde, S., O’BrieN, P. J. \& LI, J. H. 2001. The Hengshan and Wutai complexes of northern China: lower and upper crustal domains of a Neoarchaean to Palaeoproterozoic magmatic arc and significance for the evolution of the North China Craton. In 4th International Archaean Symposium 2001, Extended Abstracts (ed. K. F. Cassidy), p. 327. AGSO, Geoscience Australia, Record 37.

KröNer, A., Wilde, S. A., O’Brien, P. J., LI, J. H., PASSCHIER, C. W., WALTE, N. P. \& LiU, D. Y. 2005b. Field relationships, geochemistry, zircon ages and evolution of a Neoarchaean to Palaeoproterozoic lower crustal section in the Hengshan Terrain of Northern China. Acta Geologica Sinica 79, 605-29.

Kröner, A., Wilde, S. A., ZhaO, G. C., O'Brien, P. J., SUN, M., LIU, D. Y., WAN, Y. S., LIU, S. W. \& GUO, J. H. 2006. Zircon geochronology of mafic dykes in the Hengshan Complex of northern China: evidence for late Palaeoproterozoic rifting and subsequent high-pressure event in the North China Craton. Precambrian Research 146, 45-67.

Kusky, T. M. \& LI, J. H. 2003. Palaeoproterozoic tectonic evolution of the North China Craton. Journal of Asian Earth Sciences 22, 383-97.

LI, J. H. \& QIAN, Q. L. 1991. A study on the Longquanguan shear zone in the northern part of the Taihang Mountains. Shanxi Geology 6, 17-29 (in Chinese).

LI, J. H. \& QIAN, X. L. 1994. Early Precambrian crustal Evolution of the Hengshan Metmaorphic Terrain, North China Craton. Tianyuan: Shanxi Science and Technology Press.

Li, J. L., WanG, K. Y., Wang, C. Q., LiU, X. H. \& ZhaO, Z. Y. 1990. An Early Proterozoic collision belt in the Wutaishan area, China. Scientia Geologica Sinica 25, $1-11$ (in Chinese).

LI, S. Z., HAN, Z. Z., LIU, Y. J. \& YANG, Z. S. 2001a. Geological and geochemical constraints on Palaeoproterozoic pre-Orogenic deep processes in the Jiao-Liao Block. Scientia Geologica Sinica 36, 184-94 (in Chinese).

LI, S. Z., HAN, Z. Z., LIU, Y. J., YANG, Z. S. \& MA, R. $2001 b$. Regional metamorphism of the Liaohe Group: implications for continental dynamics. Geological Review 47, 9-18 (in Chinese).

LI, S. Z., HaO, D. F., ZhaO, G. C., Sun, M., HAN, Z. Z. \& GuO, X. Y. 2004a. Geochemical features and origin of Dandong granite. Acta Petrologica Sinica 20, 1417-23.

LI, S. Z., ZhaO, G. C., Sun, M., Wu, F. Y., HaO, D. F., HAN, Z. Z., LUO, Y. \& XIA, X. P. 2005. Deformational history of the Palaeoproterozoic Liaohe Group in the Eastern Block of the North China Craton. Journal of Asian Earth Sciences 24, 654-69.

Li, S. Z., ZhaO, G. C., Sun, M., Wu, F. Y., LiU, J. Z., HaO, D. F., HAN, Z. Z. \& LUO, Y. 2004b. Mesozoic, not Palaeoproterozoic SHRIMP U-Pb zircon ages of two Liaoji granites, Eastern Block, North China Craton. International Geology Review 46, 162-76.

LiU, D. Y., PAge, R. W., Compston, W. \& WU, J. S. 1985. U$\mathrm{Pb}$ zircon geochronology of Neoarchaean metamorphic rocks in the Taihangshan-Wutaishan area, North China. Precambrian Research 27, 85-109.

LIU, S. W., LI, J. H., PAN, Y. M., ZhANG, J. \& LI, Q. G. $2002 a$. An Archaean continental block in the Taihangshan and Hengshan regions: Constraints from geochronology and geochemistry. Progress in Natural Science 12, 568-76.

LiU, S. W., Liang, H. H., ZhaO, G. C., HuA, Y. G. \& Jian, A. H. 2000. Isotopic chronology and geological events of Precambrian complex in the Taihangshan region. Science in China (Series D) 43, 386-93. 
LiU, S. W., PAN, P. M., LI, J. H., LI, Q. G. \& ZHANG, J. $2002 b$. Geological and isotopic geochemical constraints on the evolution of the Fuping Complex, North China Craton. Precambrian Research 117, 41-56.

LiU, S. W., PAN, Y. M., XIE, Q. L., Zhang, J. \& LI, Q. G. 2004a. Archaean geodynamics in the Central Zone, North China Craton: Constraints from geochemistry of two contrasting series of granitoids in the Fuping and Wutai Complexes. Precambrian Research 130, 229-49.

LIU, S. W., PAN, Y. M., XIE, Q. L., ZhaNG, J. \& LI, Q. G. 2005. Geochemistry of the Palaeoproterozoic Nanying Granitoid Gneisses: constraints on the tectonic setting of the Central Zone, North China Craton. Journal of Asian Earth Sciences 24, 643-58.

LIU, S. W., SHEN, Q. H. \& GENG, Y. S. 1996. Metamorphic evolution of two types of garnetiferous granulites from the northwestern Hebei Province and P-T estimation by the Gibbis method. Acta Petrologica Sinica 12, 261-75.

LiU, S. W., SHU, G. M., PAN, Y. M. \& DANG, Q. N. $2004 b$. Electronic microprobe dating and metamorphic age of Wutai Group, Wutai Mountains. Geological Journal of Chinese Universities 10, 356-63 (in Chinese with English abstract).

LiU, S. W., ZhaO, G. C., Wilde, S. A., Shu, G. M., Sun, M., LI, Q. G., TIAN, W. \& ZHANG, J. 2006. Th-U-Pb monazite geochronology of the Lüliang and Wutai Complexes: constraints on the tectonothermal evolution of the Trans-North China Orogen. Precambrian Research 148, 205-25.

Lu, X. P., Wu, F. Y., Guo, J. H., Wilde, S. A., Yang, J. H., LiU, X. M. \& ZhANG, X. O. 2006. Zircon U-Pb geochronological constraints on the Palaeoproterozoic crustal evolution of the Eastern block in the North China Craton. Precambrian Research 146, 138-64.

LuO, Y., Sun, M., ZhaO, G. C., LI, S. Z., Xu, P., Ye, K. \& XIA, X. P. 2004. LA-ICP-MS U-Pb zircon ages of the Liaohe Group in the Eastern Block of the North China Craton: constraints on the evolution of the Jiao-Liao-Ji Belt. Precambrian Research 134, 349-71.

Nelson, B. K. \& DePaolO, D. J. 1985. Rapid production of continental crust 1.7-1.9 b.y. ago: $\mathrm{Nd}$ and $\mathrm{Sr}$ isotopic evidence from the basement of the North American midcontinent. Geological Society of America Bulletin 96, 746-54.

O'Brien, P. J., Walte, N. \& Li, J. H. 2005. The petrology of two distinct granulite types in the Hengshan Mts, China, and tectonic implications. Journal of Asian Earth Sciences 24, 615-27.

PASschier, C. W. \& WAlte, N. 2002. Deformation of the Hengshan Complex. In A Neoarchaean to Palaeoproterozoic Lower to Upper Crustal Section in the Hengshan-Wutaishan Area of North China (eds A. Kröner, G. C. Zhao, S. A. Wilde, M. G. Zhai, C. W. Passchier, M. Sun, J. H. Guo, P. J. O’Brien \& N. Walte), pp. 11-13. Guidebook for Penrose Conference Field Trip, September 2002. Beijing: Chinese Academy of Sciences.

PEARCE, J. A. 1976. Statistical analysis of major element patterns in basalts. Journal of Petrology 17, 15-43.

Peng, P., Zhai, M. G., Zhang, H. F. \& GuO, J. H. 2005. Geochronological constraints on the Palaeoproterozoic evolution of the North China craton: SHRIMP zircon ages of different types of Mafic dikes. International Geology Review 47, 492-508.

Polat, A., Herzberg, C., Munker, C., Rodgers, R., Kusky, T. M., LI, J. H., Fryer, B. \& Delaney, J. 2006.
Geochemical and petrological evidence for a suprasubduction zone origin of NeoArchaean $(2.55-2.50 \mathrm{Ga})$ peridotites, central orogenic belt, North China Craton. Geological Society of America Bulletin 118, 771-84.

Polat, A., Kusky, T. M., LI, J. H., FrYer, B., Kerrich, R. \& PATRICK, K. 2005. Geochemistry of NeoArchaean (ca. 2.55-2.50 Ga) volcanic and ophiolitic rocks in the Wutaishan greenstone belt, central orogenic belt, North China craton: Implications for geodynamic setting and continental growth. Geological Society of America Bulletin 117, 1387-99.

REN, J. S. 1980. Tectonic evolution of China. Beijing: Science Press.

RIVERS, T. \& CORRIGAN, D. 2000. Convergent margin on southeastern Laurentia during the Mesoproterozoic: tectonic implications. Canadian Journal of Earth Sciences 37, 359-83.

ŞENGÖR, A. M. C., NATAL'In, B. A. \& Burtman, V. S. 1993. Evolution of the Altaid tectonic collage and Paleozoic crustal growth in Eurasia. Nature 364, 299-307.

SuN, D. Z. 1997. The Jiangxian Group. In Paleoproterozoic Era (ed. Committee of Lexicon of China Stratigraphy), pp. 28-9. Beijing: Geological Publishing House.

Sun, D. Z., Hu, W. X., TANG, M. \& ZHAO, F. Q. 1993. The geochronological framework and crustal structures of Precambrian basement in the Zhongtiaoshan area. Beijing: Geological Publishing House.

Sun, M., Armstrong, R. L. \& LAmbert, R. St. J. 1992. Petrochemistry and $\mathrm{Sr}, \mathrm{Pb}$ and $\mathrm{Nd}$ isotopic geochemistry of Early Precambrian rocks, Wutaishan and Taihangshan areas, China. Precambrian Research 56, 1-31.

Tian, W., LiU, S. W., LiU, C. H., Yu, S. Q., LI, Q. G. \& WANG, Y. R. 2005. SHRIMP U-Pb zircon ages and geochemistry of the Sushui TTG gneisses in the Zhongtiao Complex and their geological significance. Progress in Natural Science 15, 1476-84.

TIAN, Y. Q. 1991. Geology and Mineralization of the WutaiHengshan Greenstone Belt. Taiyuan: Shanxi Science and Technology Press.

TIAN, Y. Q., LiANG, Y. F., FAN, S. K., ZHU, B. Q. \& CHEN, L. W. 1992. Geochronology and Nd isotopic geochemistry of the Hengshan Complex. Geochemica Sinica 3, 25563.

Wan, Y. S., Wilde, S. A., LiU, D. Y., YAnG, C. X., SonG, B. \& YIN, X. Y. 2006. Further evidence for $\sim 1.85 \mathrm{Ga}$ metamorphism in the central Zone of the north China Craton: SHRIMP U-Pb dating of zircons from metamorphic rocks in the Lushan area, Henan Province. Gondwana Research 9, 189-97.

WANG, K. Y., LI, J. L., HaO, J., Li, J. H. \& ZhOU, S. P. 1996. The Wutaishan mountain belt within the Shanxi Province, Northern China: a record of Neoarchaean collision tectonics. Precambrian Research 78, 95-103.

WANG, K. Y., LI, J. L., HAO, J., LI, J. H. \& ZHOU, S. P. 1997. Neoarchaean mafic-ultramafic rocks from the Wuatishan, Shanxi Province: a possible ophiolite melange. Acta Petrologica Sinica 13, 139-51.

WANG, K. Y., LI, J. L. \& LIU, L. Q. 1991. Petrogenesis of the Fuping grey gneisses. Acta Geologica Scientia 11, 254-67.

WANG, K. Y., Wang, Z., Yu, L., FAn, H., Wilde, S. A. \& CAWOOD, P. A. 2001. Evolution of Archaean greenstone belt in the Wutaishan region, North China: constraints from SHRIMP zircon $\mathrm{U}-\mathrm{Pb}$ and other geochronological and isotope information. In 4th International $\mathrm{Ar}$ chaean Symposium 2001, Extended Abstracts (ed. K. F. 
Cassidy), pp. 104-5. AGSO, Geoscience Australia, Record 37.

WANG, Y. J., FAN, W. M., Zhang, Y. \& GuO, F. 2003. Structural evolution and ${ }^{40} \mathrm{Ar}-{ }^{39} \mathrm{Ar}$ dating of the Zanhuang metamorphic domain in the North China Craton: constraints on Palaeoproterozoic tectonothermal overprinting. Precambrian Research 122, 159-82.

WANG, Y. J., FAN, W. M., ZHANG, Y. H., GUO, F., ZHANG, H. \& PenG, P. 2004a. Geochemical Ar-40/Ar-39 geochronological and $\mathrm{Sr}-\mathrm{Nd}$ isotopic constraints on the origin of Palaeoproterozoic mafic dikes from the southern Taihang Mountains and implications for the ca. 1800 Ma event of the North China Craton. Precambrian Research 135, 55-77.

WANG, Z. H., WILDE, S. A., WANG, K. Y. \& YU, L. J. $2004 b$. A MORB-arc basalt-adakite association in the $2.5 \mathrm{Ga}$ Wutai greenstone belt: Neoarchaean magmatism and crustal growth in the North China Craton. Precambrian Research 131, 323-43.

WILDE, S. 2002. SHRIMP U-Pb zircon ages of the Wutai Complex. In A Neoarchaean to Palaeoproterozoic Lower to Upper Crustal Section in the HengshanWutaishan Area of North China (eds A. Kröner, G. C. Zhao, S. A. Wilde, M. G. Zhai, C. W. Passchier, M. Sun, J. H. Guo, P. J. O’Brien \& N. Walte). Guidebook for Penrose Conference Field Trip, September 2002, pp. 32-4. Beijing: Chinese Academy of Sciences.

Wilde, S. A., CaWood, P. \& Wang, K. Y. 1997. The relationship and timing of granitoid evolution with respect to felsic volcanism in the Wutai Complex, North China Craton. Proceedings of the 30th IGC. Precambrian Geology and Metamorphic Petrology 17, $75-88$.

WILDE, S. A., CAWOOD, P. A. \& WANG, K. Y. 1998. SHRIMP $\mathrm{U}-\mathrm{Pb}$ data of granites and gneisses in the TaihangshanWutaishan area: Implications for the timing of crustal growth in the North China Craton. Chinese Science Bulletin 43, 144.

Wilde, S. A., CAWOOD, P. A., WANG, K. Y. \& NeMchin, A. A. 2005. Granitoid evolution in the Late Archaean Wutai Complex, North China Craton. Journal of Asian Earth Sciences 24, 597-613.

Wilde, S. A., Cawood, P. A., Wang, K. Y., Nemchin, A. \& ZHAO, G. C. 2004a. Determining Precambrian crustal evolution in China: a case-study from Wutaishan, Shanxi Province, demonstrating the application of precise SHRIMP U-Pb geochronology. In Aspects of the Tectonic Evolution of China (eds J. Malpas, C. J. N. Fletcher, J. R. Ali \& J. C. Aitchison), pp. 526. Geological Society of London, Special Publication no. 226.

Wilde, S. A., ZHAO, G. C. \& Sun, M. 2002. Development of the North China Craton during the Neoarchaean and its final amalgamation at $1.8 \mathrm{Ga}$; some speculations on its position within a global Palaeoproterozoic Supercontinent. Gondwana Research 5, 85-94.

Wilde, S. A., ZhaO, G. C., WANG, K. Y. \& Sun, M. 2004b. First precise SHRIMP U-Pb zircon ages for the Hutuo Group, Wutaishan: further evidence for the Palaeoproterozoic amalgamation of the North China Craton. Chinese Science Bulletin 49, 8390.

Windley, B. F., AleXeiev, D., Xiao, W. J., Kröner, A. \& BADARCH, G. 2007. Tectonic models for accretion of the Central Asian Orogenic Belt. Journal of the Geological Society, London 164, 31-47.
Windley, B. F., KrÖNER, A., GuO, J. H., QU, G. S., LI, Y. Y. \& ZHANG, C. 2002. Neoproterozoic to Paleozoic geology of the Altai orogen, NW China: New zircon age data and tectonic evolution. Journal of Geology 110, 719-37.

WU, C. H., Li, H. M., ZhonG, C. T. \& ZhUO, Y. C. 2000. TIMS U-Pb single zircon ages for the orthogneisses and paragneisses of Fuping Complex. Progress in Precambrian Research 23, 130-9.

Wu, C. H. \& Zhong, C. T. 1998. The Palaeoproterozoic SW-NE collision model for the central North China Craton. Progress in Precambrian Research 21, 28-50 (in Chinese).

Wu, F. Y., ZHAO, G. C., Wilde, S. A. \& SUN, D. Y. 2005. Nd isotopic constraints on crustal formation in the North China Craton. Journal of Asian Earth Sciences 24, 524 46.

Wu, J. S., GenG, Y. S., XU, H. F., Jin, L. G., He, S. Y. \& Sun, S. W. 1989. Metamorphic geology of the Fuping Group. Tianjin Institute of Geology Bulletin 19, 1-213.

XIA, X. P., Sun, M., ZHAO, G. C. \& LuO, Y. 2006a. LAICP-MS U-Pb geochronology of detrital zircons from the Jining Complex, North China Craton and its tectonic significance. Precambrian Research 144, 199-212.

Xia, X. P., Sun, M., ZhaO, G. C., Wu, F. Y., Xu, P., ZHANG, J. H. \& LUO, Y. 2006b. U-Pb and Hf isotopic study of detrital zircons from the Wulashan khondalites: Constraints on the evolution of the Ordos Terrane, Western Block of the North China Craton. Earth and Planetary Science Letters 241, 581-93.

Xia, X. P., SUN, M., ZhaO, G. C., WU, F. Y., Zhang, J., He, Y. H., XU, P. \& ZHANG, J. G. 2006c. U-Pb and Hf Isotope Study of Detrital Zircons from the Wanzi Supracrustals: constraints on the tectonic setting and evolution of the Fuping Complex, Trans-North China Orogen. Acta Geologica Sinica 80, 844-63.

Xiao, W. J., Windley, B. F., HaO, J. \& Zhai, M. G. 2003. Accretion leading to collision and the Permian Solonker suture, Inner Mongolia, China: Termination of the central Asian orogenic belt. Tectonics 22, 1069, doi: $10.1029 / 2002$ TC001484.

XiaO, W. J., Windley, B. F., LiU, D. Y., Jian, P., LiU, C. Z., YUAN, C. \& SUN, M. 2005. Accretionary tectonics of the Western Kunlun Orogen, China: A Paleozoicearly Mesozoic, long-lived active continental margin with implications for the growth of southern Eurasia. Journal of Geology 113, 687-705.

XiaO, W. J., Zhang, L. C., QIN, K. Z., Sun, S. \& LI, J. L. 2004. Paleozoic accretionary and collisional tectonics of the Eastern Tianshan (China): Implications for the continental growth of Central Asia. American Journal of Science 304, 370-95.

YU, J. H., WANG, D. Z. \& WANG, X. Y. 1997. Ages of the Lüliang Group and its main metamorphism in the Lüliang Mountains, Shanxi: evidence from single-grain zircon U-Pb ages. Geological Review 43, 403-8.

YUAN, G. P. \& ZHANG, R. Y. 1993. The structural environment of the paleo-rift in the Wutai greenstone belt. Shanxi Geology 8, 21-8.

Zhai, M. G., Bian, A. G. \& ZhaO, T. P. 2000. The amalgamation of the supercontinent of North China Craton at the end of Neo-Archaean and its breakup during late Palaeoproterozoic and Mesoproterozoic. Science in China (Series D) 43, 219-32.

ZHAI, M. G., GUO, J. H. \& YAN, Y. H. 1992. Discovery and preliminary study of the Archaean high-pressure 
granulites in the North China. Science in China 12B, 1325-30.

Zhang, J. S., DiRKs, H. G. M. \& PAsschier, C. W. 1994. Extensional collapse and uplift in a polymetamorphic granulite terrain in the Archaean and Palaeoproterozoic of North China. Precambrian Research 67, 37-57.

Zhang, J., ZhaO, G. C., SUN, M., Wilde, S. A., LI, S. Z. \& LIU, S. W. 2006. High-pressure mafic granulites in the Trans-North China Orogen: Tectonic significance and age. Gondwana Research 9, 349-62.

ZHAO, G. C. 2001. Palaeoproterozoic assembly of the North China Craton. Geological Magazine 138, 87-91.

Zhao, G. C., CaO, L., Wilde, S. A., Sun, M. \& LI, S. Z. 2006. Implications based on the first SHRIMP U-Pb zircon dating on Precambrian granitoid rocks in North Korea. Earth and Planetary Science Letters 251, 36579.

Zhao, G. C., Cawood, P. A. \& LU, L. Z. 1999. Petrology and $\mathrm{P}-\mathrm{T}$ history of the Wutai amphibolites: implications for tectonic evolution of the Wutai Complex, China. Precambrian Research 93, 181-99.

ZhaO, G. C., CaWoOd, P. A., Wilde, S. A. \& LU, L. Z. 2000a. Metamorphism of basement rocks in the Central Zone of the North China Craton: implications for Palaeoproterozoic tectonic evolution. Precambrian Research 103, 55-88.

ZHAO, G. C., CAWOOD, P. A., WILDE, S. A. \& LU, L. Z. $2001 a$. High-pressure granulites (retrograded eclogites) from the Hengshan Complex, North China Craton: Petrology and Tectonic implications. Journal of Petrology 42, $1141-70$

ZhaO, G. C., CAWood, P. A., Wilde, S. A. \& Sun, M. $2002 a$. Review of global 2.1-1.8 Ga orogens: implications for a
pre-Rodinia supercontinent. Earth-Science Reviews 59, 125-62.

ZhaO, G. C., Sun, M., Wilde, S. A. \& LI, S. Z. 2004. A Paleo-Mesoproterozoic supercontinent: Assembly, growth and breakup. Earth-Science Reviews 67, 91-123.

ZhaO, G. C., Sun, M., Wilde, S. A. \& Li, S. Z. 2005. Neoarchaean to Palaeoproterozoic evolution of the North China Craton: key issues revisited. Precambrian Research 136, 177-202.

ZhaO, G. C., Wilde, S. A., CaWood, P. A. \& LU, L. Z. 1998. Thermal evolution of the Archaean basement rocks from the eastern part of the North China Craton and its bearing on tectonic setting. International Geology Review 40, 706-21.

ZhaO, G. C., Wilde, S. A., CaWood, P. A. \& LU, L. Z. 1999. Tectonothermal history of the basement rocks in the western zone of the North China Craton and its tectonic implications. Tectonophysics 310, 37-53.

ZHAO, G. C., WiLdE, S. A., CAWOOD, P. A. \& LU, L. Z. $2000 b$. Petrology and $\mathrm{P}-\mathrm{T}$ path of the Fuping mafic granulites: Implications for tectonic evolution of the central zone of the North China Craton. Journal of Metamorphic Geology 18, 375-91.

Zhao, G. C., Wilde, S. A., CaWood, P. A. \& Sun, M. $2001 b$. Archaean blocks and their boundaries in the North China Craton: lithological, geochemical, structural and $\mathrm{P}-\mathrm{T}$ path constraints and tectonic evolution. Precambrian Research 107, 45-73.

ZhaO, G. C., WiLdE, S. A., CAWOOD, P. A. \& SUN, M. $2002 b$. SHRIMP U-Pb zircon ages of the Fuping Complex: implications for accretion and assembly of the North China Craton. American Journal of Science 302, 191226. 